\title{
Nanoscale
}

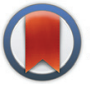

CrossMark

\&lick for updates

Cite this: Nanoscale, 2015, 7, 19764

\section{Recent advances in transition-metal dichalcogenide based nanomaterials for water splitting}

\author{
Fengmei Wang, $\dagger^{a, b}$ Tofik Ahmed Shifa, $\dagger^{a, b}$ Xueying Zhan, ${ }^{a}$ Yun Huang, ${ }^{a, b}$ Kaili Liu, ${ }^{a, b}$ \\ Zhongzhou Cheng, ${ }^{a}$ Chao Jiang ${ }^{\mathrm{C}}$ and Jun $\mathrm{He}^{*^{\mathrm{a}}}$
}

\begin{abstract}
The desire for sustainable and clean energy future continues to be the concern of the scientific community. Researchers are incessantly targeting the development of scalable and abundant electro- or photocatalysts for water splitting. Owing to their suitable band-gap and excellent stability, an enormous amount of transition-metal dichalcogenides (TMDs) with hierarchical nanostructures have been extensively explored. Herein, we present an overview of the recent research progresses in the design, characterization and applications of the TMD-based electro- or photo-catalysts for hydrogen and oxygen evolution. Emphasis is given to the layered and pyrite-phase structured TMDs encompassing semiconducting and metallic nanomaterials. Illustrative results and the future prospects are pointed out. This review will provide the readers with insight into the state-of-the-art research progresses in TMD based nanomaterials for water splitting
\end{abstract}

Received 28th September 2015 Accepted 5th November 2015

DOI: $10.1039 / c 5 n r 06718 a$

www.rsc.org/nanoscale
The combustion of fossil fuels produces large quantities of pollutant gases, such as nitrogen oxides, carbon oxides and sulfur oxides and so on. For quite a long time, researchers have been trying to find renewable, clean and carbon-neutral energy sources in order to stave off catastrophic climate change. Hydrogen, having largest energy density, can be combusted to liberate energy without any harmful side products. A mol of hydrogen can produce $286 \mathrm{~kJ}$ energy through reaction with oxygen $\left(2 \mathrm{H}_{2}(\mathrm{~g})+\mathrm{O}_{2}(\mathrm{~g}) \rightarrow 2 \mathrm{H}_{2} \mathrm{O}(\mathrm{l})\left(\Delta H=-286 \mathrm{~kJ} \mathrm{~mol}^{-1}\right)\right)$. More importantly, hydrogen can be utilized as an energy carrier $^{1-3}$ to store solar and electric energy via forming the chemical bond between two hydrogen atoms. Thus, the ever

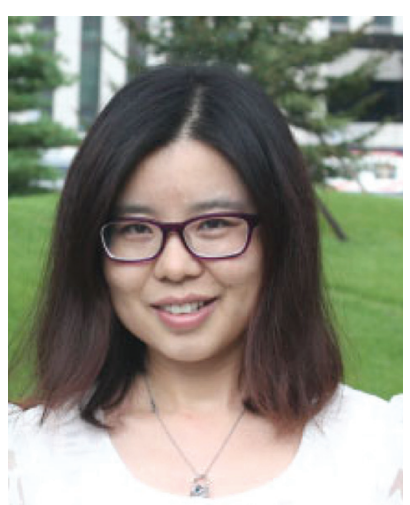

Fengmei Wang
Fengmei Wang received her BSC degree in Applied Chemistry from Hunan University in 2012. She is currently a PhD candidate under the supervision of Prof. Jun He at National Center for Nanoscience and Technology (NCNST), China. Her major research interests include synthesis and development of transition-metal chalcogenides for electrocatalytic and photocatalytic water splitting.

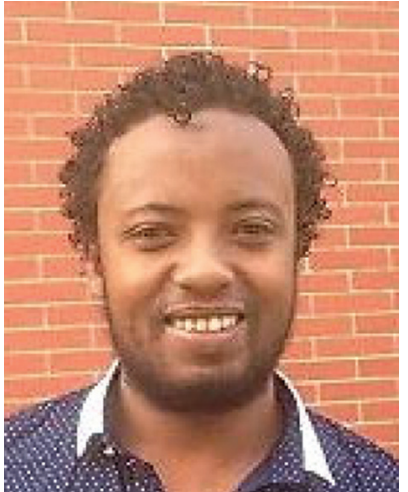

Tofik Ahmed Shifa
Tofik Ahmed received his BSc degree in Applied Chemistry from Arbaminch University, Ethiopia in 2007. He then obtained his MSc. degree in Analytical Chemistry from Haramaya University, Ethiopia in 2011. Currently, he is pursuing a PhD degree under the supervision of Prof. Jun He at National Center for Nanoscience and Technology (NCNST), Beijing, China. His research focuses on the controllable synthesis of layered transition-metal dichacogenides for water splitting. 
greenest approach to generate hydrogen can conspicuously solve the problem of the energy crisis. In industry, hydrogen is produced by steam reforming of natural gas $\left(\mathrm{CH}_{4}(\mathrm{~g})+\mathrm{H}_{2} \mathrm{O}(\mathrm{g})\right.$ $\left.\rightarrow \mathrm{CO}(\mathrm{g})+3 \mathrm{H}_{2}(\mathrm{~g})\right){ }^{4}$ Nevertheless, this process usually consumes fossil fuels and releases $\mathrm{CO}_{2}$ gas. As a matter of fact, water is a well acknowledged resource for being the most plentiful supply of hydrogen that can be used to produce hydrogen via electrocatalytic $c^{5,6}$ or photocatalytic ${ }^{7-11}$ water splitting. The water splitting reaction, however, is an uphill process through which $237 \mathrm{~kJ} \mathrm{~mol}^{-1}$ of energy is required. In an electrochemical system, the external circuit usually provides the energy to realize splitting water including hydrogen evolution reaction (HER) and oxygen evolution reaction (OER). ${ }^{12,13}$ In this regard, the electrocatalysis can accelerate the rate of a chemical reaction through providing an alternative mechanism involving a different transition state and lower activation energy to reduce the electrochemical overpotentials. ${ }^{4,14-17}$ Precious noble metals or alloys, such as platinum (Pt), palladium (Pd), ruthenium ( $\mathrm{Ru}$ ) and iridium (Ir), have traditionally been the most efficient catalysts for water splitting in acid or alkaline electrolyte ${ }^{18,19}$ but the low abundance and consequently high cost of such metals hinder their long-term viability. Therefore, efficient electrocatalysts comprised of earth-abundant materials for HER and OER are crucial for the development of water-splitting devices.

To date, earth-abundant transition-metal dichalcogenides (TMDs) with general formula $\mathrm{MX}_{2}(\mathrm{M}=$ transition metal; $\mathrm{X}=$ chalcogen) including two-dimensional (2D) layered materials, such as $\mathrm{MoS}_{2}, \mathrm{WS}_{2}, \mathrm{MoSe}_{2}, \mathrm{WSe}_{2}, \mathrm{TaS}_{2}$ and $\mathrm{TiS}_{2}$ etc., and pyrite phase structured TMDs $\left(\mathrm{CoSe}_{2}, \mathrm{CoS}_{2}, \mathrm{NiS}_{2}, \mathrm{NiSe}_{2}, \mathrm{FeS}_{2}\right.$ and so on) are being developed at rapid pace for water splitting. ${ }^{20-27}$ In particular, featuring toward the $2 \mathrm{D}$ morphology and atomic thickness, these $2 \mathrm{D}$ layered $\mathrm{MX}_{2}$ nanosheets present some unusual physical, chemical or elec-

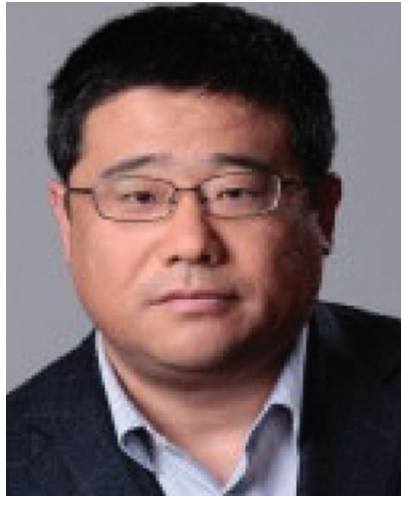

Jun He
Prof. He received his $P h D$ in Semiconductor Physics from the Institute of Semiconductors, Chinese Academy of Sciences (CAS), in 2003. Then he worked as a postdoctoral fellow at Applied Physics Department of Technische Universiteit Eindhoven, Netherlands, and Material Department of University of California, Santa Babara, USA. From 2007 to 2010, he worked at California NanoSystem Institute (CNSI), University of California, Los Angeles, USA, as a research scientist. He joined the "100Talents" Program of CAS in Nov. 2010 and became a Full Professor of NCNST since then. Up to date, He is the author or coauthor of more than 60 refereed papers. The total SCI citation times exceed 1000. tronic properties compared to their bulk counterparts. ${ }^{6,24,28-32}$ Typically, each layer of them comprises three atomic layers with covalently bonded $\mathrm{X}-\mathrm{M}-\mathrm{X}$ and adjacent $\mathrm{MX}_{2}$ layers are coupled by weak van der Waals forces to form bulk crystals. $^{33,34}$ In 2005, Jens Nørskov's group explored the HER mechanism on different catalysts through density functional theory (DFT) calculations. ${ }^{35}$ They found that the Gibbs free energy $(\Delta G)$ of $\mathrm{MoS}_{2}$ nanoparticles for HER is comparable to $\mathrm{Pt}$, suggesting the potential HER property of $\mathrm{MoS}_{2}$. Subsequently, the edge of $\mathrm{MoS}_{2}$ nanosheet was experimentally confirmed to be electrochemically active. ${ }^{36}$ As such, experimental and computational studies demonstrate other 2D TMDs ( $\mathrm{WS}_{2}, \mathrm{MoSe}_{2}, \mathrm{WSe}_{2}, \mathrm{TaS}_{2}$ and $\mathrm{TiS}_{2}$ ) are electrocatalytically active for $\mathrm{H}_{2}$ production. Unlike X-M-X layers in 2D layered TMDs, the family of cubic pyrite-type or orthorhombic macarsite-type structured TMDs shows the metal atoms are octahedrally bonded to adjacent chalcogen atoms. These TMD materials have been previously used as catalysts for ORR, ${ }^{37-39}$ HER $^{40,41}$ and OER. ${ }^{42-45}$ The partial filling of the $e_{g}$ band was proved to contribute to their superior electrocatalytic activity.

From the perspective of photocatalysis, theoreticians ${ }^{46-48}$ put forward the promising photocatalytic ability of segmental semiconducting TMDs for solar water splitting. As it is obvious, the band gap of $\mathrm{MoS}_{2}$, typical TMD, increases dramatically when the thickness reduces from a few layers to an ideal monolayer. The single-layer $\mathrm{MoS}_{2}$ has the potential to split water under visible light. However, scalable synthesis of monolayer $\mathrm{MoS}_{2}$ nanosheets remains challenging. In line with this, these materials usually serve as cocatalysts in the photocatalytic system. ${ }^{49-53}$ Engineering advanced nanocomposites by designing hybrids with other materials is the most fascinating approach to overcome the shortcoming of individual counterparts as well as optimize their performance in photocatalytic water splitting. Moreover, the recent success in the synthesis of these TMD materials via various solution/vaporprocess and scalable techniques offers great opportunities to construct functional nanostructures for hydrogen and oxygen evolution.

In this review, we briefly trace the strategies in TMD based nanomaterials applied in electrocatalytic and photocatalytic water splitting and give an overview of a clear understanding of basic knowledge, challenges and opportunities to point out the state-of-the-art in this field. We summarize major fabrication methods, describe some of the progresses and resulting achievements. Finally, we discuss the future prospects and afford some insights into the research directions in this area.

\section{Synthesis of TMD based nanomaterials}

The synthesis of TMDs with layer controllability and large-area uniformity is an indispensable requirement for practical application in water splitting. Meanwhile, exposing catalytically active sites of the TMDs should be considered by engineering the preparation process. Thus, great efforts have been devoted 
to generate high-quality TMD layers with a unique size, controllable thickness and excellent electronic structures. Nowadays, chemical exfoliation (top-down approach), solvothermal and chemical vapour deposition (both of which are bottom-up approaches) are frequently reported methods. Of these, individually or as a combination can be used to grow best performing TMDs. The following sections focus on the details of chemical exfoliation method, solvo(hydro)thermal method and chemical vapor deposition method.

\subsection{Chemical exfoliation method}

Tracing back to the discovery of graphene, ${ }^{54}$ the emergence of new physical properties are evident when a bulk crystal of macroscopic dimensions is thinned down to monolayer. ${ }^{55}$ This renders the opportunity to engineer the electronic structure. The bulk, unexfoliated, state of TMDs such as $\mathrm{MoS}_{2}, \mathrm{WS}_{2}{ }^{56,57}$ exist in Semiconducting $2 \mathrm{H}$ phases (trigonal prismatic) where most of the active sites are not exposed. This hinders their application in various fields. The $\mathrm{M}-\mathrm{X}-\mathrm{M}$ layers in TMD which are bound to each other by weak van der Waals forces can be exfoliated to further exploit the potential advantages. ${ }^{58,59}$

Liquid-phase exfoliation is advantageous over simple mechanical exfoliation owing to the formation of novel hybrid and composite materials; yet it is time consuming, environmentally unfriendly, and it is difficult to find appropriate solvents apart from resulting in structural deformation. ${ }^{60}$ Sonication assisted liquid-phase exfoliation ${ }^{61}$ appears to be a plausible route in so far the problem with solvent compatibility is addressed by using mixtures of solvents.

The product, however, is small in yield. The selection of solvents is very critical, and it is usually made based on Hansen solubility parameters (HSP) theory ${ }^{62,64,65}$ that would ultimately result in the minimum energy of exfoliation. The HSP theory predicts how well a material will dissolve in a given solvent or mixture of solvents and form a solution. Such prediction is made by taking three energy forms, namely the energy from dispersion forces $\left(\delta_{\mathrm{d}}\right)$, the energy from dipolar intermolecular forces $\left(\delta_{\mathrm{p}}\right)$ and the energy from hydrogen bonds $\left(\delta_{\mathrm{h}}\right)$ as governing parameters into consideration. Assuming these three parameters as co-ordinates in a three dimensional space called Hansen space, the interaction radius $\left(R_{\mathrm{a}}\right)$ is calculated as an indicator to the distance between two molecules (eqn (1)). Accordingly, the nearer the two molecules are in this three-dimensional space; the more likely they are to dissolve in each other.

$$
\begin{aligned}
R_{\mathrm{a}}= & {\left[4\left(\delta_{\mathrm{D}, \text { solv. }}-\delta_{\mathrm{D}, \text { solu. }}\right)^{2}+\left(\delta_{\mathrm{P}, \text { solv. }}-\delta_{\mathrm{P}, \text { solu. }}\right)^{2}\right.} \\
& \left.\left.+\left(\delta_{\mathrm{H}, \text { solv. }}-\delta_{\mathrm{H}, \text { solu }}\right)^{2}\right)\right]^{0.5}
\end{aligned}
$$

Thus, the smaller $R_{\mathrm{a}}$ is the higher the expected solubility. It has been suggested that nanomaterials are most effectively dispersed in solvents with matching surface energy. ${ }^{61}$ Most often, a mixed solvent leads to a better result. In this case, each of the three HSP parameters can be obtained as a linear sum of the entities according to the following eqn (2).

$$
\delta_{\text {mix }}=\sum \phi_{n, \text { component }} \delta_{n, \text { component }}
$$

where $\phi$ is the volume fraction for each composition. For instance, Zhou et al. ${ }^{62}$ demonstrated that a mixture of ethanol and water can be designed to give high solubility for efficient exfoliation of $\mathrm{MoS}_{2}$ and $\mathrm{WS}_{2}$. Optimum ethanol-water ratio was set to achieve a stable suspension that does not precipitate under ambient conditions. Different ethanol-water mixtures have different $R_{\mathrm{a}}$ value which in turn is related to the dispersion concentration. It is obvious from Fig. 1a that $45 \mathrm{vol} \%$ ethanol-water is optimum condition for the $\mathrm{MoS}_{2}$ case and therefore the smallest calculated $R_{\mathrm{a}}$ is resulted in the highest dispersion concentration (Fig. 1b). As such, the exfoliated nanomaterials in Fig. 1c exist as thin sheets with sizes varying from $100 \mathrm{~nm}$ to several micrometers and more importantly the hexagonal lattices of the obtained $\mathrm{MoS}_{2}$ nanosheets were not damaged during the mixed-solvent exfoliation process as evidenced from Fig. 1d. In a similar fashion, the case of $\mathrm{WS}_{2}$ also follows the same trend at $35 \mathrm{vol} \%$ ethanol-water (Fig. 1e-h). In so far the application in HER is concerned, Li ion intercalation method is the most frequently reported technique that leads to high yield of the catalyst. ${ }^{23,63,66-70}$ It mostly involves the reduction of butyllithium reagent which results in expansion of the lattice at the van der Waals gap thereby destabilizing the thermodynamically favored semiconducting $2 \mathrm{H}$ phase and eventually transforming to octahedral $1 \mathrm{~T}$ phase. Fig. 1i depicts the role of lithium intercalation chemistry deriving the phase transition from $2 \mathrm{H}$ to $1 \mathrm{~T}$ of $\mathrm{WS}_{2}$ nanosheet as reported by Lukowski and coworkers. ${ }^{63}$ The high crystallinity of the multilayered structure was confirmed from the lattice-resolved highresolution transmission electron microscopy (Fig. 1j and k) and the $2 \mathrm{H}-\mathrm{WS}_{2}$ feature was indexed from the selected area electron diffraction (SAED) pattern (inset of Fig. 1k). After intercalation, layered material was chemically exfoliated with excess water, resulting in individual $1 \mathrm{~T}-\mathrm{WS}_{2}$ nanosheets. The high density of exposed edge sites was evidenced from the inset of Fig. 11 revealing the more disordered and open structures. Though there are still untransformed $2 \mathrm{H}-\mathrm{WS}_{2}$ nanosheets, the presence of $1 \mathrm{~T}-\mathrm{WS}_{2}$ nanosheet is confirmed from the superlattice structure observed in the SAED pattern (red circles inset of Fig. 1m). This was corroborated by Voiry et $a .^{23}$ for the strained and chemically exfoliated $\mathrm{WS}_{2}$ nanosheet. Such strain and zigzag-like local lattice distortion as shown in Fig. 1n for the $1 \mathrm{~T}$ phase of $\mathrm{WS}_{2}$ is brought about by the Li intercalation and ultimately facilitated the HER process. Upon annealing, the $1 \mathrm{~T}$ phase relaxed back to the thermodynamically favored $2 \mathrm{H}$ phase (Fig. 10). High yield (>80\%) of metallic $1 \mathrm{~T}$ phase $\mathrm{MoS}_{2}$ nanosheets can be obtained using a solvent-free intercalation method. For example, Voiry and coworkers ${ }^{70}$ used lithium borohydride $\left(\mathrm{LiBH}_{4}\right)$ to exfoliate bulk $\mathrm{MoS}_{2}$ powder into single layered nanosheets from the solid-state intercalation reaction at $300{ }^{\circ} \mathrm{C}$ under argon environment. The yield in their case is significantly higher than the $n$-butyllithium exfoliated $\operatorname{MoS}_{2}(\sim 50 \%)^{71}$ prepared at room temperature. Moreover, electrochemical lithiation is also another method reported to be advantageous over simple chemical intercalation by virtue of its relatively facile, roomtemperature operation and provides high yield of products of 

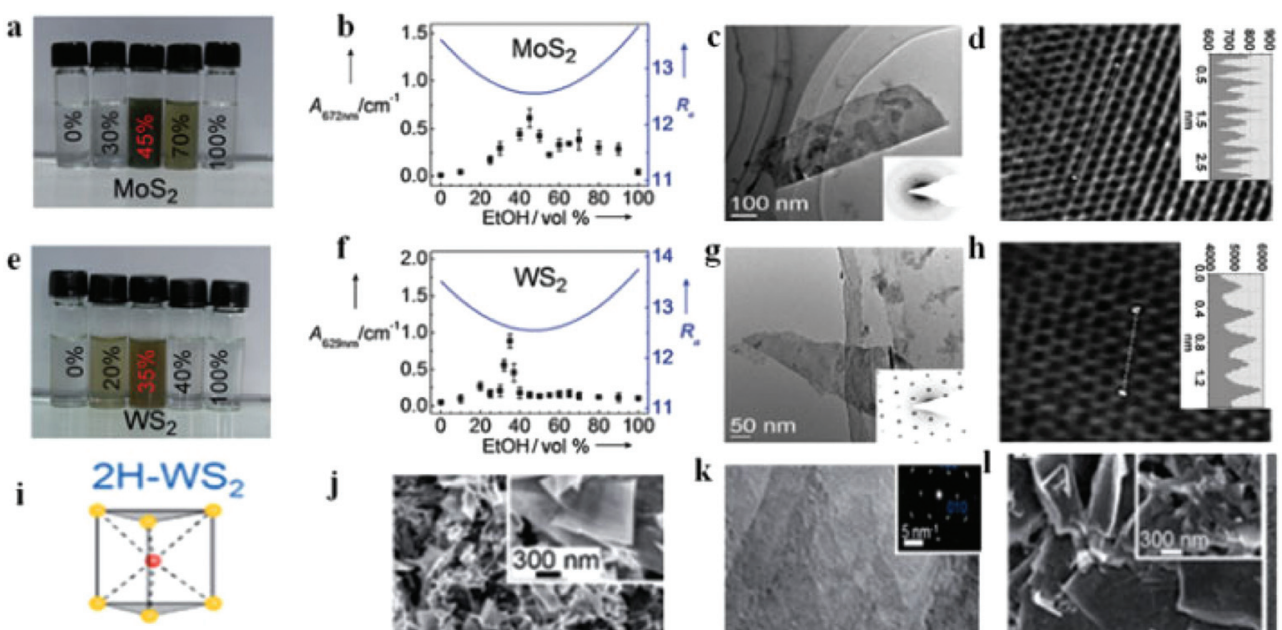

$\mathbf{j}$
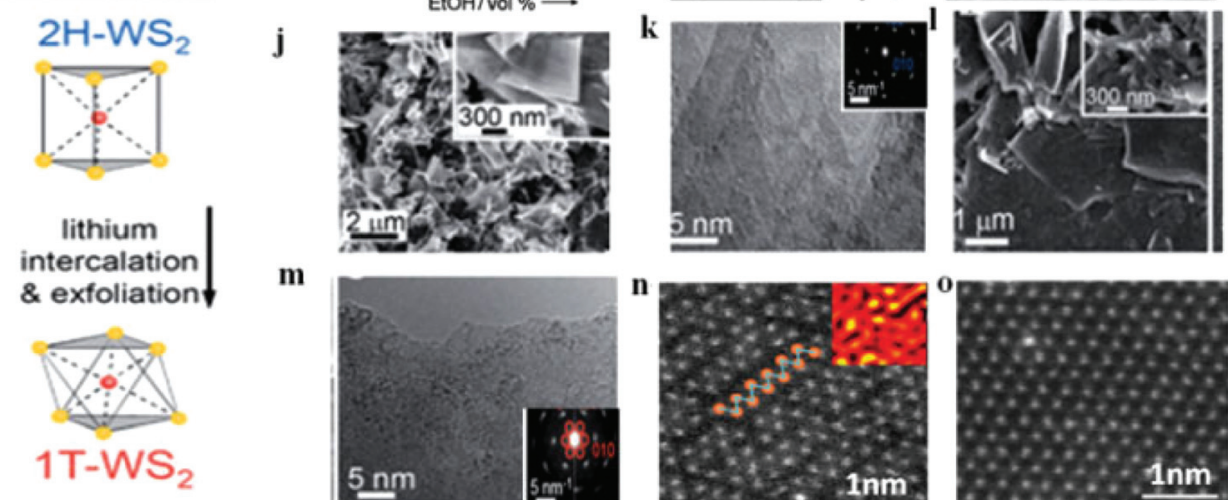

m
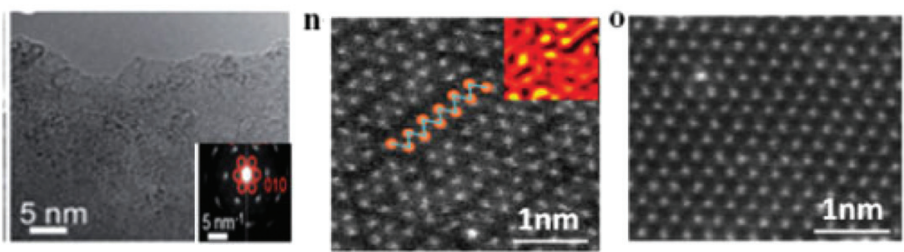

Fig. 1 Photographs of $\mathrm{MoS}_{2}$ (a) and $\mathrm{WS}_{2}$ (e) dispersions in various ethanol-water mixtures. The absorbance of the MoS $\mathrm{S}_{2}(\mathrm{~b})$ and WS $\mathrm{S}_{2}$ ( $\mathrm{f}$ ) suspensions in ethanol-water mixtures with different composition are shown as dots, and the calculated $R_{\mathrm{a}}$ values as solid lines. HRTEM image of MoS 2 . (c) and $W_{2}$ (g). Atomic resolution HRTEM image of $M_{0} S_{2}(d)$ and $W_{2}$ (h) (a-g, reproduced with permission from ref. 62. Copyright 2011 John Wiley \& Sons, Inc.). (i) Simple lithium intercalation chemistry as used to drive the phase transformation from the $2 \mathrm{H}-$ to the $1 \mathrm{~T}-\mathrm{WS} \mathrm{S}_{2}$ polymorph. (j-k) Top-down SEM and HRTEM images of the as-grown $2 \mathrm{H}-\mathrm{WS}_{2}$ nanostructures on W foil. (l-m) Top-down SEM and HRTEM images of chemically exfoliated WS 2 nanosheets cast on graphite ( $\mathrm{i}-\mathrm{m}$, reproduced with permission from ref. 63. Copyright 2014 The Royal Society of Chemistry). (n-o) High-resolution STEM images of an as-exfoliated $\mathrm{WS}_{2}$ monolayer showing regions exhibiting the $1 \mathrm{~T}$ superlattice $(\mathrm{n})$ and $2 \mathrm{H}$ structures (o) (reproduced with permission from ref. 23. Copyright 2013 Macmillan Publishers Limited).

single layer. In this particular method, the bulk TMD is set as cathode and lithium foil as anode in a Li-ion battery setup. ${ }^{72}$ The galvanostatic discharge process with optimized conditions induces the lithium intercalation. Zhang's group ${ }^{72}$ effectively optimized the cutoff voltage to prepare few-layered $\mathrm{NbSe}_{2}$ and $\mathrm{WSe}_{2}$ nanosheets. In their related report, single layers of highyield $\mathrm{MoS}_{2}, \mathrm{WS}_{2}, \mathrm{TiS}_{2}, \mathrm{TaS}_{2}$ and $\mathrm{ZrS}_{2}$, were successfully achieved. ${ }^{73}$ This method eases the control of optimum amount of $\mathrm{Li}$ ion to be inserted as too low or too much $\mathrm{Li}$ insertion brings about serious deterioration in the final product. Upon lithium intercalation, charge impurities are vulnerable as a result of $\mathrm{Li}$ ions in TMDs. In fact, this kind of impurity is detrimental in electronics and especially in $2 \mathrm{D}$ materials. ${ }^{74}$ It is therefore important to remove the excess charge from the surface by a simple method such as treating them with iodine dissolved in acetonitrile. ${ }^{70}$

\subsection{Hydro(solvo) thermal method}

Hydrothermal method refers to purely heterogeneous reactions using water as media in a sealed steel pressure container with Teflon liners. It is capable of synthesizing advanced inorganic materials in high yield. ${ }^{75}$ Typically, the temperature of the hydrothermal process is in the range of $100-200{ }^{\circ} \mathrm{C}$ and the pressure is generated autogenously. ${ }^{76}$ The moderate temperatures together with the advantage of fast reaction kinetics and short processing time render it as a promising route to prepare homogeneous products in large scale effectively and economically. ${ }^{77}$ Based on those merits, as one type of advanced inorganic materials, TMD based nanomaterials with different shapes are widely fabricated by hydrothermal method recently. ${ }^{78-81}$ For example, it has been possible to successfully synthesize $\mathrm{MoS}_{2}$ nanoflowers, ${ }^{78} \mathrm{MoS}_{2}$ microspheres, ${ }^{79} \mathrm{CoS}_{2}$ nanowires, ${ }^{80}$ and $\mathrm{CoS}_{2}$ nanoparticles, ${ }^{83}$ which all display great potential for application of water splitting. To further improve their catalytic activity, composites that hybrid TMDs with other conductive or porous materials have been synthesized. $\mathrm{MoS}_{2}$ nanosheets have successfully been grown on carbon fiber (CF), ${ }^{84,85}$ graphene oxide (GO), ${ }^{88}$ and Mo meshes. ${ }^{82}$ As conductive materials, $\mathrm{CF}$ and GO can facilitate electron transportation. Furthermore, the $\mathrm{MoS}_{2}$ nanostructure grown on these three-dimensional (3D) CF, GO and Mo meshes can expose more active sites. These design principles can also be applied to other members of TMDs. ${ }^{89,90}$ For instance, $\mathrm{CoSe}_{2}$ nanowires array on carbon cloth $\left(\mathrm{CoSe}_{2} \mathrm{NW} / \mathrm{CC}\right)^{89}$ and nitrogen-doped graphene supported $\mathrm{CoSe}_{2}$ nanobelt composite (NG-CoSe composite) were prepared. ${ }^{44}$ Fig. $2 \mathrm{a}$ is a schematic illustration 


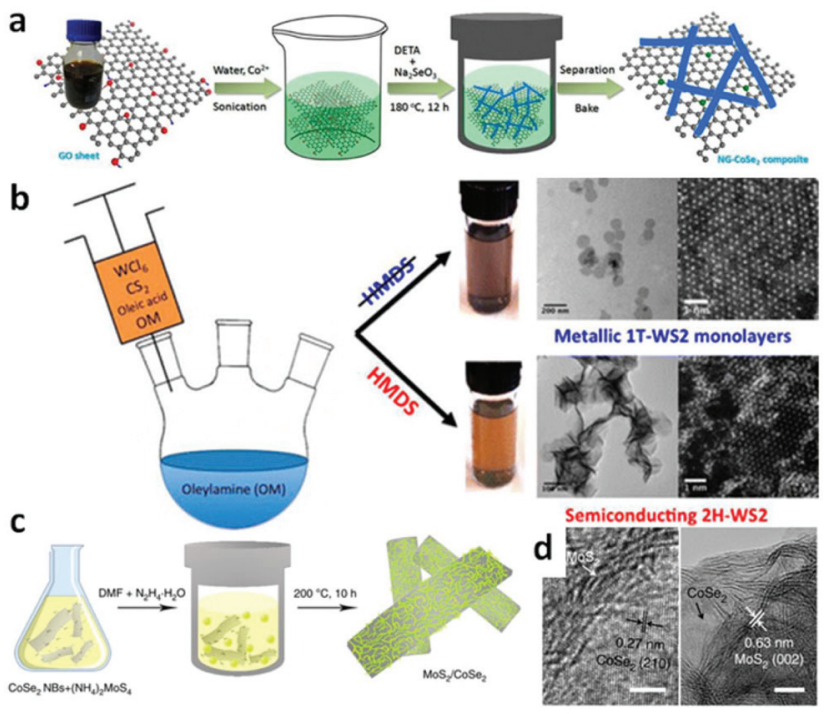

Fig. 2 (a) Schematic illustration of the formation of nitrogen-doped graphene supported $\mathrm{CoSe}_{2}$ nanobelt composite (reproduced with permission from ref. 44. Copyright 2014 American Chemical Society). (b) Schematic solvothermal process to synthesize $W_{2}$ nanosheets, lowmagnification TEM images and HAADF-HRSTEM images of $1 T-W_{2}$ nanosheets and $2 \mathrm{H}-\mathrm{WS}_{2}$ nanosheets (reproduced with permission from ref. 86. Copyright 2015 American Chemical Society). (c) Schematic illustration of solvothermal synthesis with $\mathrm{CoSe}_{2} / \mathrm{DETA}$ nanobelts as substrates for preparation of $\mathrm{MoS}_{2} / \mathrm{CoSe}_{2}$ hybrid. (d) HRTEM images of $\mathrm{MoS}_{2} / \mathrm{CoSe}_{2}$ hybrid showing distinguishable microstructures of $\mathrm{MoS}_{2}$ and $\mathrm{CoSe}_{2}$ (reproduced with permission from ref. 87. Copyright 2015 Macmillan Publishers Limited).

of the hydrothermal process to fabricate $\mathrm{NG}-\mathrm{CoSe}_{2}$ hybrid composites. Typically, the mixture of $\mathrm{Co}(\mathrm{AC})_{2} \cdot \mathrm{H}_{2} \mathrm{O}$ and $\mathrm{GO}$ was sonicated for about $10 \mathrm{~min}$ followed by the addition of diethylenetriamine (DETA) and $\mathrm{Na}_{2} \mathrm{SeO}_{3}$. This new mixture was homogenized, heated to $180{ }^{\circ} \mathrm{C}$ and kept for $12 \mathrm{~h}$ to yield $\mathrm{CoSe}_{2}$ nanobelts which were anchored to the NG sheets.

Solvents other than water can also be employed accounting for the fact that organic solvents have various specific physicochemical properties such as polarity, viscosity, softness, which all can affect the solubility and transport behavior of the precursors during the synthetic process. ${ }^{91}$ The shape, size and phases of the final samples can be controlled in these solvents. ${ }^{91}$ The most important aspect of solvothermal method is that it prevents the samples from oxidizing; hence this method can be used to prepare various non-oxides, such as TMD based nanomaterials..$^{22,92-94}$ With this method, Benoit et al. have synthesized $1 \mathrm{~T}-\mathrm{WS}_{2}$ and $2 \mathrm{H}-\mathrm{WS}_{2}$ nanosheets (Fig. $2 \mathrm{~b}$ ). ${ }^{86}$ To obtain $1 \mathrm{~T}-\mathrm{WS}_{2}$ nanosheets, they degassed oleylamine (OM) in a threeneck flask for $1 \mathrm{~h}$ under vacuum at $65{ }^{\circ} \mathrm{C}$ and then heated up to $320{ }^{\circ} \mathrm{C}$ under argon atmosphere. Next, the mixture of OM, $\mathrm{WCl}_{6}$, oleic acid and $\mathrm{CS}_{2}$ was injected dropwise into the $\mathrm{OM}$ solution over a $30 \mathrm{~min}$ interval. As shown in Fig. 2b, the synthetic process of $2 \mathrm{H}-\mathrm{WS}_{2}$ nanosheets is similar, except a further procedure, adding hexamethyl disilazane (HMDS) into the OM after degassing the solution. The $1 \mathrm{~T}-\mathrm{WS}_{2}$ nanosheets are monodispersed with diameter of about $100 \mathrm{~nm}$ whereas that of the $2 \mathrm{H}-\mathrm{WS}_{2}$ nanosheets are aggregated and some of them are still flower-like. Highly scalable $\mathrm{MoS}_{2}$ nanostructures have also been fabricated on $\mathrm{CF},{ }^{95}$ and graphene ${ }^{94,96,97}$ to construct efficient electrocatalysts via this method. To prepare the $\mathrm{MoS}_{2}$ nanoparticles on reduced graphene oxide $\left(\mathrm{MoS}_{2} / \mathrm{RGO}\right)$ hybrid, ${ }^{96}\left(\mathrm{NH}_{4}\right)_{2} \mathrm{MoS}_{4}$ and graphene oxide (GO) were mixed in $\mathrm{N}, \mathrm{N}$-dimethylformamide (DMF) solvent and then the mixture was sonicated to form a clear and homogeneous solution. After heating the reaction solution at $200{ }^{\circ} \mathrm{C}$ for $10 \mathrm{~h}$, uniformly laid $\mathrm{MoS}_{2}$ nanoparticles on rGO sheets resulted with size of around $5 \mathrm{~nm}$. Other composite materials can also be synthesized by using a solvothermal method which renders the synergetic chemical coupling effects between the components making up the composites. Yu's group successfully synthesized the uniform $\mathrm{CoSe}_{2}$ nanobelts through the reaction between $\mathrm{Co}(\mathrm{Ac})_{2} \cdot \mathrm{H}_{2} \mathrm{O}$ and $\mathrm{Na}_{2} \mathrm{SeO}_{3}$ in DETA at $180{ }^{\circ} \mathrm{C}$ with lengths of several tens of micrometers and widths of from 100 to $500 \mathrm{~nm} . .^{98}$ Due to the copious amino groups on the $\mathrm{CoSe}_{2} /$ DETA surface, which serve as the nucleation sites, some other inorganic precursors can anchor on the $\mathrm{CoSe}_{2}$ /DETA to nucleate and grow under solvothermal method. For this reason, they hybridized their $\mathrm{CoSe}_{2}$ nanobelts with $\mathrm{Mn}_{3} \mathrm{O}_{4}$ nanoparticles $\left(\mathrm{Mn}_{3} \mathrm{O}_{4} / \mathrm{CoSe}_{2}\right){ }^{42} \mathrm{Ni} / \mathrm{NiO}$ nanoparticles $(\mathrm{Ni} / \mathrm{NiO} /$ $\left.\mathrm{CoSe}_{2}\right)^{99}$ and $\mathrm{MoS}_{2}$ nanosheets $\left(\mathrm{MoS}_{2} / \mathrm{CoSe}_{2}\right) \cdot{ }^{87} \mathrm{Fig}$. 2c displays a schematic illustration of solvothermal synthesis of $\mathrm{MoS}_{2} /$ $\mathrm{CoSe}_{2}$ hybrid with $\mathrm{CoSe}_{2} /$ DETA nanobelts as substrates. During the synthesis, $\mathrm{CoSe}_{2} / \mathrm{DETA}$ nanobelts and precursors were mixed and sonicated, followed by heating at $200{ }^{\circ} \mathrm{C}$ for $10 \mathrm{~h}$. From the high-resolution transmission electron microscopy (HRTEM) images of $\mathrm{MoS}_{2} / \mathrm{CoSe}_{2}$ hybrid (Fig. 2d), it can be seen that the uniform $\mathrm{MoS}_{2}$ nanosheets tightly combined with $\mathrm{CoSe}_{2}$ nanobelts. A similar procedure was employed for the synthesis of $\mathrm{Mn}_{3} \mathrm{O}_{4} / \mathrm{CoSe}_{2}{ }^{42}$ and $\mathrm{Ni} / \mathrm{NiO} / \mathrm{CoSe}_{2}{ }^{99}$ nanomaterials.

\subsection{Vapor-phase deposition method}

Vapor-phase deposition methods, including sulfurization (or selenization) reaction with metal or metal oxide, ${ }^{102-113}$ thermal decomposition of thiosalts, ${ }^{114}$ and vapor-phase transport recrystallization from powders, ${ }^{115-117}$ are extensively utilized to controllably synthesize TMDs. ${ }^{118}$ Generally, this method realizes the growth of TMD nanostructures with large scale and high quality on the different substrates for hydrogen or oxygen evolution. The superior interfaces between nanostructures and substrates are in favor of charge transportation. What's more, by adjusting the ratio of precursors, the component of products could be easily controlled to improve the physical and chemical properties. ${ }^{119,120}$ With these merits, researchers have proposed a great many projects to increase the catalytic activity of TMDs for water splitting via vapor deposition routes. One tendency is to design nanostructures with huge numbers of active sites. For example, Cui's group ${ }^{100}$ designed a rapid sulfurization/selenization process to convert Mo thin film to a distinctive structure of vertical aligned $\mathrm{MoS}_{2}$ and $\mathrm{MoSe}_{2}$ films on several kinds of substrates. As shown in Fig. 3a-e, vertical structures greatly gain the exposed edges of 


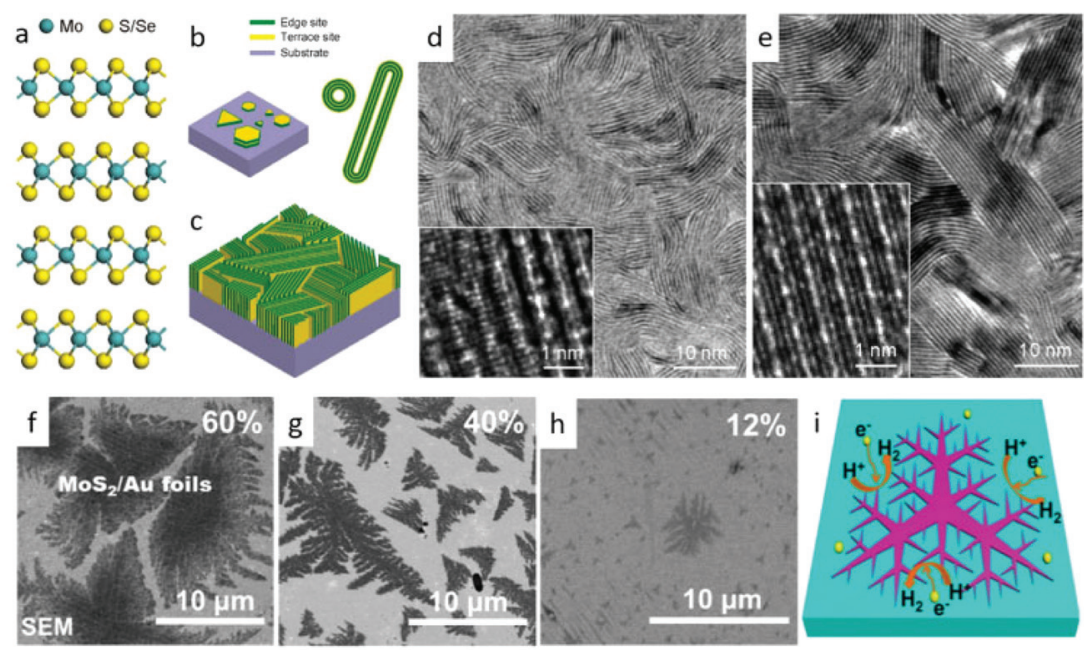

Fig. 3 (a) Layered crystal structure of molybdenum dichalcogenide. (b) Schematics of $\mathrm{MoS}_{2}$ nanoparticle with platelet-like morphology distributed on a substrate (left), and nanotubes and fullerene-like nanotubes of $\mathrm{MoS}_{2}$ and $\mathrm{MoSe}_{2}$ (right). (c) Idealized structure of edge-terminated molybdenum dichalcogenide films with the layers aligned perpendicular to the substrate, maximally exposing the edges of the layers. TEM image of a MoS $\mathrm{S}_{2}$ film (d) and a $\mathrm{MoSe}_{2}$ film (e) produced by rapid sulfurization/selenization, clearly showing exposed edges. High-resolution TEM image (inset) reveals individual layers consisting of three atomic planes in the sequence of S-Mo-S or Se-Mo-Se atomic planes (reproduced with permission from ref. 100.

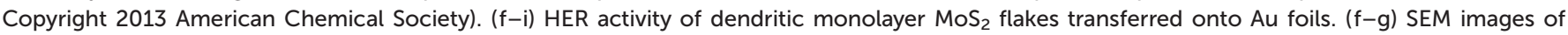
monolayer $\mathrm{MoS}_{2}$ flakes on Au foils with coverages of 60,40 and $12 \%$ and domain sizes of $\sim 10 \mu \mathrm{m}, \sim 5 \mu \mathrm{m}$ and $\sim 800 \mathrm{~nm}$ (in envelope edge length), respectively. (i) Schematic view illustrating the edges of fractal monolayer $\mathrm{MoS}_{2}$ flakes as active catalytic sites for HER (reproduced with permission from ref. 101. Copyright 2014 American Chemical Society).

$\mathrm{MoS}_{2}$ and $\mathrm{MoSe}_{2}$ nanosheets. During this growth process, the sulfurization or selenization conditions play a crucial role in determining the growth direction.

At a high temperature, the much slower rate of $\mathrm{S} / \mathrm{Se}$ atoms diffusing into the deposited Mo films than that of chemical conversion renders it as the rate-limiting process. On the other hand, the anisotropic structure of $\mathrm{MoS}_{2} / \mathrm{MoSe}_{2}$ leads to $\mathrm{S} / \mathrm{Se}$ atoms diffusion along the layers faster than that across the layers. ${ }^{100}$ These two factors jointly result in the formation of such vertical structures, which greatly gain the exposed edges. Analogously, they obtained a similar structure of $\mathrm{MoSe}_{2}$ and $\mathrm{WSe}_{2}$ films on curved and rough substrates (Si nanowires and carbon fiber paper), which exhibited significantly improved HER activity than that on the flat ones. ${ }^{123}$ Benefiting from the higher surface energy of pure Si substrate, Yang and coworkers ${ }^{21}$ realized an edge-abundant $\mathrm{MoS}_{2}$ nanobelts structure with their basal plane vertical to the substrates. Since the surface was fully covered by edge sites, a high HER efficiency was achieved. Besides the vertical ones, other nanostructures with abundant edges are synthesized via vapor deposition method. For instance, by carefully choosing single-crystal $\mathrm{SrTiO}_{3}$ as substrates, Liu's group ${ }^{101}$ developed a low-pressure chemical vapor deposition (LPCVD) approach to controllably grow dendritic monolayer $\mathrm{MoS}_{2}$. As depicted in Fig. 3f, those nanoflakes were decorated with plentiful edges, much more than that of the common triangular ones. These dendritic $\mathrm{MoS}_{2}$ nanostrucures could be transferred to Au foil for further application in water splitting. Additionally, the construction of 3D hierarchical TMD structures with large surface area is also popular. Some flexible carbon fibers or porous substrates have been used for this purpose. Recently, we successfully ${ }^{29,124}$ grew $3 \mathrm{D} \mathrm{WS}_{2}$ and $\mathrm{WSe}_{2}$ nanotubes (NTs) on carbon fibers through in situ sulfurizing/selenizing $\mathrm{WO}_{3}$ nanowires ${ }^{125}$ as depicted in Fig. 4a and b. On the basis of these results, component-controllable $\mathrm{WS}_{2(1-x)} \mathrm{Se}_{2 x}$ NTs were also synthesized to modify its physical and chemical properties. The large surface area of asgrown NTs, the layer dislocations, defects and local strains existing on the walls of the NTs (Fig. 4c) are the dominant active sites for electrocatalytic water splitting. Similar 3D hierarchical structures of other TMDs like $\mathrm{CoSe}_{2}$ and $\mathrm{MoS}_{2}$ have also been reported for being successfully synthesized with this method and applied for highly sensitive HER catalysis. ${ }^{126,127}$ Compared with carbon fibers, porous substrates are able to offer greater contact area and load with more TMD nanomaterials. Jaramillo's group ${ }^{121}$ developed a method to synthesize double-gyroid $\mathrm{MoS}_{2}$ by sulfurization of Mo films, which were electrodeposited on a silica template. After that, the silica templates were dissolved by etching with a $2 \mathrm{wt} \%$ hydrofluoric acid solution and eventually the bicontinuous inverse double-gyroid $\mathrm{MoS}_{2}$ network with a higher surface area was obtained. The schematic procedure is depicted in Fig. 4d. Although it exhibited superior activity for electrocatalysis, the absence of direct contacts between the electrodes and the catalysts may impede its further improvement of properties. Thus, it is important to grow the materials directly onto conductive porous templates. Chang et al. ${ }^{128}$ developed a method to formulate $\operatorname{MoS}_{x}(x \geq 2)$ on graphene protected $\mathrm{Ni}$ foam through the thermolysis of ammonium thiomolybdates. The 


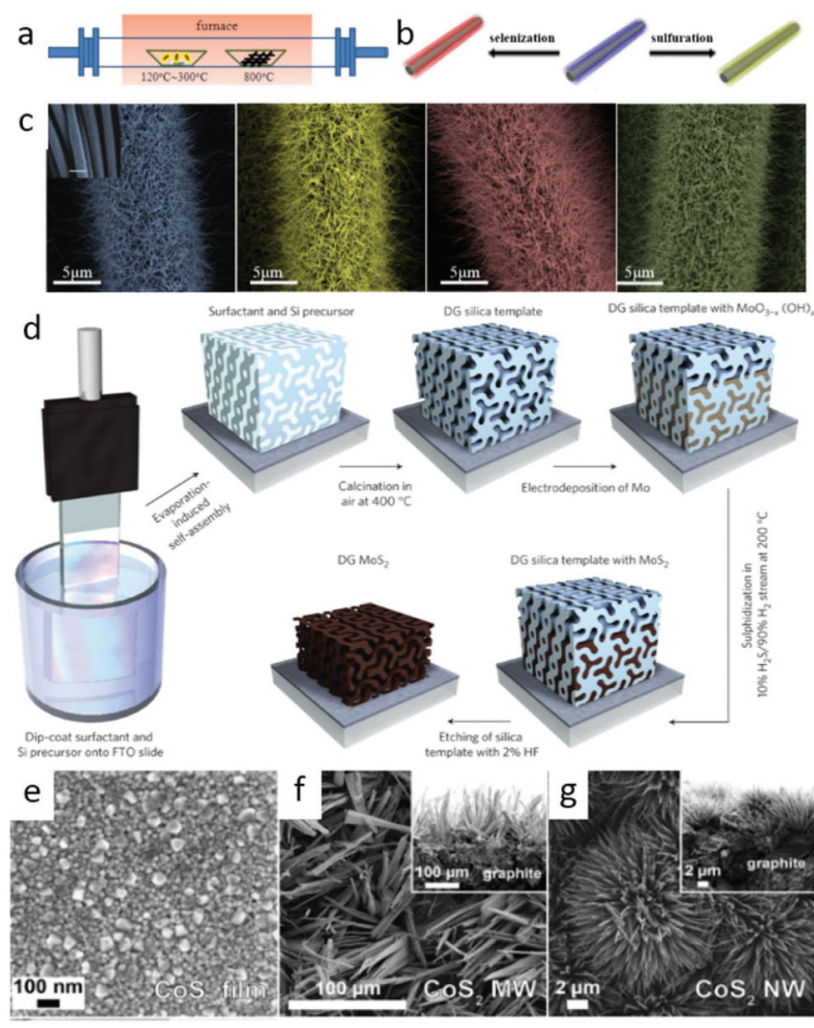

Fig. 4 (a) Schematic diagram of the experimental setup of the fabrication of $\mathrm{WO}_{3} \mathrm{NWs}$ and $\mathrm{WS}_{2}, \mathrm{WSe}_{2}$ and $\mathrm{WS}_{2(1-x)} \mathrm{Se}_{2 x} \mathrm{NTs}$. (b) Schematic diagram of $\mathrm{WO}_{3} \mathrm{NWs}$ converting to $\mathrm{WS}_{2}$ and $\mathrm{WSe}_{2} \mathrm{NTs}$ on carbon fibers by sulfuration and selenization. (c) Magnified SEM images of $\mathrm{WO}_{3} \mathrm{NWs}$, $\mathrm{WS}_{2}, \mathrm{WSe}_{2}$ and $\mathrm{WS}_{2(1-x)} \mathrm{Se}_{2 x} \mathrm{NTs}$ (from left to right) (reproduced with permission from ref. 29. Copyright 2014 American Chemical Society). (d) Synthesis procedure and structural model for mesoporous $\mathrm{MoS}_{2}$ with a double-gyroid (DG) morphology (reproduced with permission from ref. 121. Copyright 2012 Macmillan Publishers Limited). SEM images of (e) a polycrystalline $\mathrm{CoS}_{2}$ film, (f) $\mathrm{CoS}_{2} \mathrm{MWs}$ and (g) $\mathrm{CoS}_{2}$ NWs on graphite (with cross-sectional SEM images inset in the corresponding panels) (reproduced with permission from ref. 122. Copyright 2014 American Chemical Society).

$\mathrm{MoS}_{x} /$ graphene/3D Ni foam structure showed excellent HER efficiency. Similarly, Tan et al. utilized 3D nanoporous gold as supports to synthesize monolayer $\mathrm{MoS}_{2}$ films and applied them for HER. ${ }^{129}$

Some pyrite phase structured TMDs, such as $\mathrm{CoSe}_{2}, \mathrm{CoS}_{2}$ and $\mathrm{NiS}_{2}$ nanostructures have been achieved by vapor deposition method. $\mathrm{CoSe}_{2}$ nanoparticles on carbon cloth were pre- pared through seleniding cobalt oxide nanoparticles by Cui's group. ${ }^{41}$ Faber et al. ${ }^{122}$ reported that $\mathrm{CoS}_{2}$ film, nanowires and microwires were synthesized on graphite or glass substrates via sulfurizing Co films, $\beta-\mathrm{Co}(\mathrm{OH})_{2}$ microwires and $\mathrm{Co}(\mathrm{OH})$ $\left(\mathrm{CO}_{3}\right)_{0.5} \cdot x \mathrm{H}_{2} \mathrm{O}$ nanowires in $\mathrm{S}$ vapor atmosphere. Fig. $4 \mathrm{e}-\mathrm{g}$ display the SEM images of as-grown $\mathrm{CoS}_{2}$ films, nanowires and microwires on graphite. All the results verified the $\operatorname{CoX}_{2}(X=S$, Se) as high performance electrocatalyst and their potential for large-scale application of renewable energy. Besides, other earth-abundant metal pyrites $\left(\mathrm{FeS}_{2}, \mathrm{NiS}_{2}\right)$ and their alloys have also been synthesized and examined as promising alternative electrocatalysts for both HER and ORR. ${ }^{40,130,131}$ In brief, vapor deposition is an effective method to produce TMDs nanostructures with designed morphology and components, which are promising for application of water splitting.

\section{Properties and application in water splitting}

\subsection{Electrocatalytic water splitting}

As an advanced technology for clean energy conversion, water electrolysis is a subject of both extensive fundamental and utilitarian studies. The core of this technology is a series of electrochemical processes. Typically, in the electrocatalysis of water, the HER and OER processes occur at the cathode and anode of an electrolytic cell to produce gaseous molecular hydrogen and oxygen, respectively (eqn (3)). ${ }^{132}$ These processes are as follows:

$$
\begin{aligned}
& \text { Cathode }: 2 \mathrm{H}^{+}+2 \mathrm{e}^{-} \rightarrow \mathrm{H}_{2} \\
& \text { Anode }: 2 \mathrm{H}_{2} \mathrm{O} \rightarrow \mathrm{O}_{2}+4 \mathrm{H}^{+}+4 \mathrm{e}^{-}
\end{aligned}
$$

The hydrogen evolution reaction (HER) is perhaps the most studied electrochemical reaction and is of prominence for applications such as energy storage via $\mathrm{H}_{2}$ production. It has been well established ${ }^{96,133}$ that HER proceeds via two successive steps: discharge of proton to give adsorbed $\mathrm{H}$ atom (Volmer reaction) followed by either a combination of two $\mathrm{H}$ atoms (Tafel reaction) or combination of an adsorbed hydrogen atom with a proton and an electron (Heyrovsky reaction). These different kinds of steps of $\mathrm{HER}^{134,135}$ are involved depending on the nature of the electrode and media of the electrolyte under investigation (Table 1). To be a good HER catalyst, it should have neither too weakly nor too strongly adsorbed $\mathrm{H}^{*}$ on to $\mathrm{M}$, having an optimal free energy of $\mathrm{H}$ adsorption close to zero. ${ }^{136}$

Table 1 Mechanism of HER catalysis in acidic and basic media ${ }^{a}$

\begin{tabular}{lll}
\hline Processes & Acidic media & Basic media \\
\hline Volmer reaction & $\mathrm{H}^{+}+\mathrm{M}+\mathrm{e}^{-} \rightarrow \mathrm{M}-\mathrm{H}^{*}$ & $\mathrm{H}_{2} \mathrm{O}+\mathrm{M}^{+}+\mathrm{e}^{-} \rightarrow \mathrm{M}^{-} \mathrm{H}^{*}+\mathrm{OH}^{-}$ \\
Heyrovsky reaction & $\mathrm{M}-\mathrm{H}^{*}+\mathrm{H}^{+}+\mathrm{e}^{-} \rightarrow \mathrm{M}+\mathrm{H}_{2}$ & $\mathrm{M}-\mathrm{H}^{*}+\mathrm{H}_{2} \mathrm{O}+\mathrm{e}^{-} \rightarrow \mathrm{M}^{-} \mathrm{OH}^{-}+\mathrm{H}_{2}$ \\
Tafel reaction & $2 \mathrm{M}-\mathrm{H}^{*} \rightarrow 2 \mathrm{M}+\mathrm{H}_{2}$ & $2 \mathrm{M}-\mathrm{H}^{*} \rightarrow 2 \mathrm{M}+\mathrm{H}_{2}$
\end{tabular}

${ }^{a} \mathrm{M}$ denotes the electrode surface. 
Unlike HER, anodic OER, as an indispensible half reaction involved in water splitting, includes four proton-coupled electron transfers and oxygen-oxygen bond formation, which is much more kinetically sluggish and challenging. In acidic conditions, two water molecules are oxidized and separated into four protons $\left(\mathrm{H}^{+}\right)$and one oxygen molecule $\left(\mathrm{O}_{2}\right)$ through losing four electrons (eqn (4)). Likewise, the oxidation of hydroxide ions takes place in alkaline solution producing $\mathrm{O}_{2}$ and $\mathrm{H}_{2} \mathrm{O}$ with the same number of electrons being evolved (eqn (5)).

$$
\left.2 \mathrm{H}_{2} \mathrm{O} \rightarrow 4 \mathrm{H}^{+}+\mathrm{O}_{2}+4 \mathrm{e}^{-} \text {(in acidic electrolyte }\right)
$$

or

$$
4 \mathrm{OH}^{-} \rightarrow 2 \mathrm{H}_{2} \mathrm{O}+\mathrm{O}_{2}+4 \mathrm{e}^{-} \text {(in basic electrolyte) }
$$

Eqn (4) and (5) suggest that the generation of an $\mathrm{O}_{2}$ molecule requires a transfer of four electrons. However, the multiple electron transfer at same time is not kinetically favorable. Some results reveal that the OER involves multiple steps with one electron transfer per step. ${ }^{137}$ The four possible reaction mechanisms in basic media are listed in Table 2.

Generally, HER and OER obey the Butler-Volmer model even with very high overpotentials. During electrolysis, the potential $(E)$ where reaction takes place is different from Nernstian potential $\left(E_{\mathrm{r}}\right)$ and the difference between the two potentials corresponds to the extra energy than thermodynamically expected to drive the reaction. This potential is called the overpotential ( $\eta$, eqn (6)).

$$
\eta=E-E_{\mathrm{r}}
$$

In line with this, potential of interest (onset potential, or potential at certain current density), exchange current density, and Tafel slope are the most frequently reported parameters to evaluate HER catalysts. These parameters can be extracted from the current-potential relationship curve, particularly known as the polarization curve. The potential where the first catalytic current observed is called the onset potential, above which the current increases rapidly. More often, a specific potential corresponding to unambiguous current density is employed as a catalyst performance measurement.

Table 2 The possible OER mechanisms in basic media ${ }^{a}$

\begin{tabular}{ll}
\hline Mechanism I & Mechanism II \\
\hline $1.2 \mathrm{OH}^{-} \rightarrow 2 \mathrm{OH}+2 \mathrm{e}^{-}$ & $1.2 \mathrm{OH}^{-} \rightarrow 2 \mathrm{OH}+2 \mathrm{e}^{-}$ \\
$2.2 \mathrm{OH}+2 \mathrm{OH}^{-} \rightarrow 2 \mathrm{O}^{-}+2 \mathrm{H}_{2} \mathrm{O}$ & $2.2 \mathrm{OH}+2 \mathrm{OH}^{-} \rightarrow 2 \mathrm{O}^{-}+2 \mathrm{H}_{2} \mathrm{O}$ \\
$3.2 \mathrm{O}^{-} \rightarrow 2 \mathrm{O}+2 \mathrm{e}^{-}$ & $3.2 \mathrm{O}^{-}+2 \mathrm{MO}_{x} \rightarrow 2 \mathrm{MO}_{x+1}+2 \mathrm{e}^{-}$ \\
$4.2 \mathrm{O} \rightarrow \mathrm{O}_{2}$ & $4.2 \mathrm{MO}_{x+1} \rightarrow 2 \mathrm{MO}_{x}+\mathrm{O}_{2}$ \\
\hline Mechanism III & Mechanism IV \\
\hline $1.4 \mathrm{OH}^{-}+\mathrm{M} \rightarrow 4 \mathrm{M}-\mathrm{OH}+4 \mathrm{e}^{-}$ & $1.2 \mathrm{OH}^{-} \rightarrow 2 \mathrm{OH}+2 \mathrm{e}^{-}$ \\
2. $4 \mathrm{M}-\mathrm{OH} \rightarrow 2 \mathrm{MO}+2 \mathrm{M}+2 \mathrm{H}_{2} \mathrm{O}$ & $2.2 \mathrm{OH}_{2} \mathrm{OH}^{-} \rightarrow 2 \mathrm{H}_{2} \mathrm{O}_{2}^{-}$ \\
$3.2 \mathrm{MO} \rightarrow 2 \mathrm{M}+\mathrm{O}_{2}$ & $3.2 \mathrm{H}_{2} \mathrm{O}_{2}^{-} \rightarrow \mathrm{O}_{2}^{-}+2 \mathrm{H}_{2} \mathrm{O}$ \\
& $4.2 \mathrm{O}_{2}^{-} \rightarrow \mathrm{O}_{2}+2 \mathrm{e}$ \\
$a_{*} \mathrm{M}$ denotes the electrode surface. &
\end{tabular}

In this respect, the potential required to afford a current density of $10 \mathrm{~mA} \mathrm{~cm}^{-2}$ is usually reported as it corresponds to the cost competitive photoelectrochemical water splitting efficiency of $12.3 \% .{ }^{138}$ Other equally important parameters are the Tafel slope and the exchange current density. The Tafel equation $^{133}$ represents the commonest range of overpotential and current densities providing information about kinetics of charge transfer process vis-à-vis the additional voltage required, with units usually expressed as $\mathrm{mV}$ per decade $\left(\mathrm{mV} \mathrm{dec}^{-1}\right)$ of current. The relationship between current and potential was exploited and given by Butler-Volmer equation (eqn (7))

$$
I=A j_{\mathrm{o}}\left\{\exp \left[\frac{\alpha_{\mathrm{a}} n F}{R T}\right]\left(E+E_{\mathrm{eq}}\right)-\exp \left[-\frac{\alpha_{\mathrm{c}} n F}{R T}\right]\left(E-E_{\mathrm{eq}}\right)\right\}
$$

where $A$ is the electrode active surface area, $\alpha$ is the charge transfer coefficient. At considerable overpotential on the electrode surface, the equation simplifies to Tafel equation (eqn (8)):

$$
\eta=a-b \log j
$$

where $b$ is Tafel's slope, gives information on the mechanism of the electrode reaction and $j$ is the current density given by eqn (9):

$$
j=\frac{I}{\text { electrode area }}
$$

The exchange current density can be extracted from the Tafel curve, revealing the flow of charge or electron at equilibrium. It normally assumes a very low value due to the unavailability of net current at equilibrium signifying the extent of oxidation or reduction that may occur. The lower the exchange current density signifies the higher the overvoltage that must be applied to create a significant net current flow. In general, low overpotential, high exchange current density and small Tafel slope are characteristics of good electrocatalysts.

In the past few years, thanks to the remarkable advances in the nanotechnology and computational chemistry, TMDs have gained widespread attention for the design of novel electrocatalysts for HER and OER due to their unique chemical and physical properties. Herein, TMD based electrocatalysts are highlighted for hydrogen and oxygen evolution in an electrolytic cell.

3.1.1. HER catalyzed by TMD based nanomaterials. In the subsequent sections, the HER performances of layered TMDs (focusing on molybdenum and tungsten dichalcogenide based electrocatalysts) and pyrite phase TMDs (focusing on iron, cobalt and nickel dichalcogenide based electrocatalysts) will be surveyed with particular attention vested on the strategies to substantiate a better performance.

3.1.1.1. Molybdenum dichalcogenide based electrocatalysts. Following the discovery of graphene, molybdenum based dichalcogenides ${ }^{138-140}$ are the most widely investigated materials owing to their exciting prospects in HER catalysis among all TMD based nanomaterials. Inspired by the effective 
catalysis ability of hydrogenases and nitrogenases for hydrogen evolution reaction, Hinnemann et al. theoretically made the biomimetic speculation of Mo based materials to be active in HER. ${ }^{35}$ The comparison among the free energies $\left(\Delta G_{\mathrm{H}}\right)$ of atomic hydrogen bonding to the catalyst on different materials with that on the common enzymes in Fig. 5a shows that materials that form strong bonds ( $\mathrm{Ni}$ and $\mathrm{Mo}$ ) with atomic $\mathrm{H}$ or those that do not bind to atomic hydrogen $(\mathrm{Au})$ should be excluded from the expectation to be good HER catalysts. However, bearing a resemblance to the enzymes, $\mathrm{MoS}_{2}$ is a promising candidate for replacing Pt owing to the existence of active site on the edges. Experimentally, the electrocatalytic activity $^{36}$ linearly correlates with the length of edge sides as quantified from the scanning tunneling microscopy (Fig. 5b and c) and found to be independent of the $\mathrm{MoS}_{2}$ area coverage (Fig. 5d and e). Thus, activities from amorphous to nanocrystal $\mathrm{MoS}_{2}$ with various shapes have been reported so far and still continues to flourish with impressive advances. ${ }^{138,141}$ Furthermore, the HER activity of $\mathrm{MoS}_{2}$ is dependent on the size and number of layers, as reported by Seo et al. ${ }^{142}$ Nanoscale dimension at monolayer precision level provides increased performance. Generally, exposing much more edges and increasing the conductivity of $\mathrm{MoS}_{2}$ are the two main strategies pursued for improving its HER performance. The basal planes of $2 \mathrm{D} \mathrm{MoS}_{2}$ usually suffer from the paucity of active sites for HER. There are various strategies developed to expose active sites on such materials. ${ }^{21,121,143}$ Among them, inducing defects into the crystal structure of $\mathrm{MoS}_{2}$ can bring about additional active edge sites emanating from the cracks on $\mathrm{MoS}_{2}$ nanosheets. ${ }^{144}$ From Fig. 6a, the ultrathin nature of $\mathrm{MoS}_{2}$ nanosheets was confirmed from the TEM image. The direction of individual (100) planes of hexagonal $\mathrm{MoS}_{2}$ slightly differs from one another (Fig. $6 \mathrm{~b}$ and $\mathrm{c}$ ). This suggests the disordered atomic arrangement leading to cracking of the basal planes, and thereby formation of additional edges, and eventually resulting in the considerably high HER performance. Yang and coworkers $^{21}$ reported the HER performance of single-crystal $\mathrm{MoS}_{2}$ nanobelts. The top surface of the nanobelt is composed of vertically positioned basal plane edges. Such extreme exposure of edges make the material optically active, as it quenches all of the indirect band gap excitons, and also electrochemically active from the observed current density of $20 \mathrm{~mA} \mathrm{~cm}{ }^{-2}$ at overpotential of $170 \mathrm{mV}$ in acid electrolyte $(0.5$ $\mathrm{M} \mathrm{H}_{2} \mathrm{SO}_{4}$ ). From the report by Rui and coworkers, it is evident that enlarged spaces endowed by modifying the interlayer spacing can alter the electronic structures of the edge-terminated $\mathrm{MoS}_{2}$ colloid nanostructure, leading to improved performance. After expanding the interlayer spacing of $\mathrm{MoS}_{2}$ to $9.4 \AA$ (Fig. 6d), a fast kinetics of HER with onset potential of $103 \mathrm{mV}$ and Tafel slope of $49 \mathrm{mV} \mathrm{dec}{ }^{-1}$ was achieved. ${ }^{145}$ The report by Lewis et al. ${ }^{146}$ is also exemplary in so far exposing active site is concerned. They directly prepared porous $\mathrm{MoSe}_{2}$ film on glassy carbon electrode through an operando method. This film features better HER performance than the smooth film. Moreover, improving the conductivity of $\mathrm{MoS}_{2}$ through hybrids with other conductive materials or in atomic level is also popular to promote the HER performance. Molybdenum dichalcogenides based hybrid structures via enhancing the conductivity can increase the HER performance. For instance, $\mathrm{MoO}_{3} / \mathrm{MoS}_{2}$ core-shell nanowires, ${ }^{147} \mathrm{MoS}_{2} / 3 \mathrm{D}-\mathrm{CFs},{ }^{148}$ $\mathrm{MoS}_{2} / \mathrm{CNT}$-graphene, ${ }^{149} \mathrm{MoS}_{2} / \mathrm{N}$-doped carbon nanotube, ${ }^{150}$ $\mathrm{MoS}_{2} / \mathrm{Au},{ }^{151} \mathrm{MoS}_{2} / \mathrm{rGO},{ }^{96,152} \mathrm{MoSe}_{2} /$ graphene, $^{153} \mathrm{MoSe}_{2} /$ $\mathrm{SnO}_{2}{ }^{154}$ and $\mathrm{MoSe}_{2} / \mathrm{rGO}^{155}$ exhibited superior electrocatalytic activity in the HER relative to the single-component catalysts.
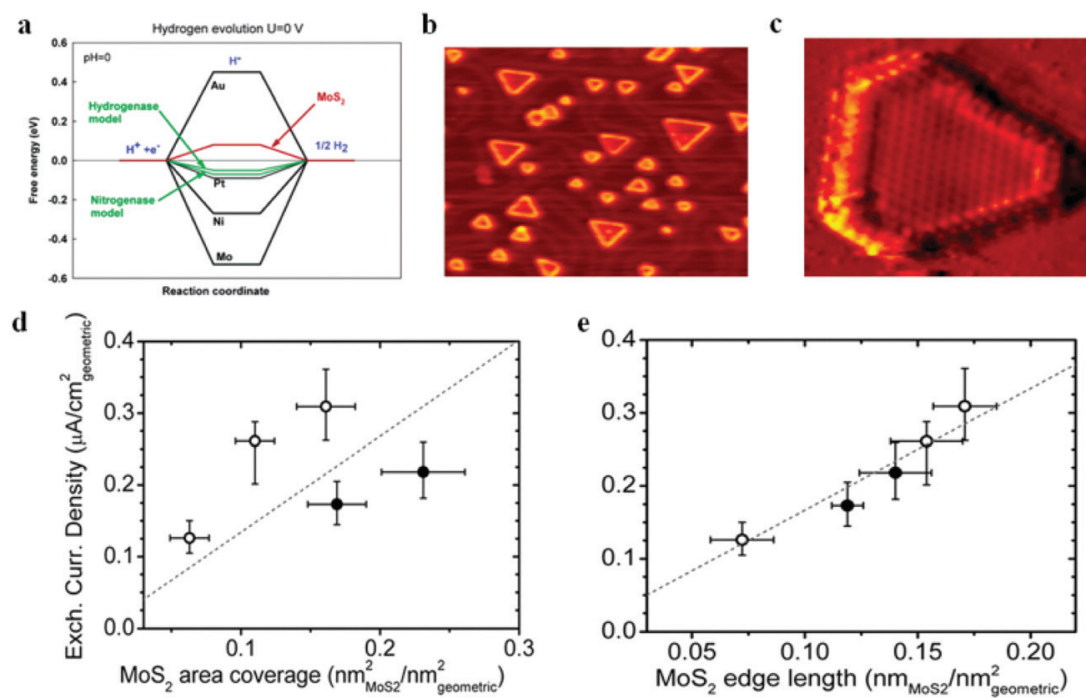

Fig. 5 (a) Calculated free energy diagram for hydrogen evolution (reproduced with permission from ref. 35. Copyright 2005 American Chemical Society). (b) STM image of high coverage $\mathrm{MoS}_{2}$ nanoparticles on $\mathrm{Au}(111)$ (c) STM image of high coverage atomically resolved MoS 2 particle. (d) Exchange current density versus $\mathrm{MoS}_{2}$ area coverage. (e) Exchange current density versus $\mathrm{MoS}_{2}$ edge length (reproduced with permission from ref. 36. Copyright 2007 American Association for the Advancement of Science). 

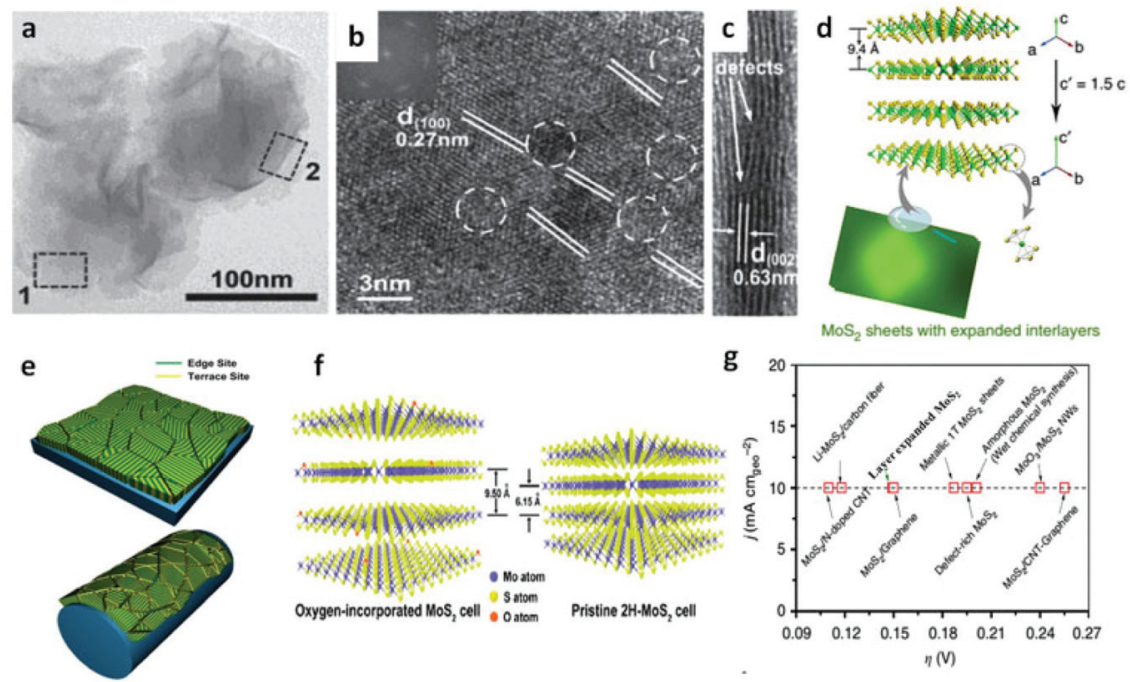

Fig. 6 (a) TEM images of defect-rich $\mathrm{MoS}_{2}$ ultrathin nanosheets. (b-c) HRTEM image areas 2 and 1, respectively [(a-c) reproduced with permission from ref. 144. Copyright 2013 John Wiley \& Sons, Inc.]. (d) Schematics of enlarged layer space in MoS $_{2}$ (reproduced with permission from ref. 22. Copyright 2015 Macmillan Publishers Limited). (e) Molybdenum chalcogenide nanofilm with molecular layers vertically standing on a flat and curved substrate. The green color represents the edge sites, and the yellow color represents the terrace sites. The edges are maximally exposed on the curved surface (reproduced with permission from ref. 123. Copyright 2013 American Chemical Society). (f) Schematic for the oxygen-incorporated $\mathrm{MoS}_{2}$ with enlarged interlayer spacing and the pristine $2 \mathrm{H}-\mathrm{MoS}_{2}$ (reproduced with permission from ref. 158. Copyright 2013 American Chemical Society). (g) Comparison of overpotential required to generate a current density of $10 \mathrm{~mA} \mathrm{~cm}{ }^{-2}$ on various MoS $_{2}$-based catalysts (reproduced with permission from ref. 22. Copyright 2015 Macmillan Publishers Limited).

The synergisms are more pronounced from a well matched vertical orientation, availing much more surface area, and ease of electron transport. This feature is typically substantiated from the report by Wang et al. who synthesized vertically oriented $\mathrm{MoS}_{2}$ on curved ${ }^{123}$ and $3 \mathrm{D}$ substrate. ${ }^{156}$ As compared to the flat substrate, the curved substrate (Fig. 6e) could increase the surface area, maximize the exposed edge sites and induce strain through expanding or squeezing the molecular layers, thereby changing the electronic structures of the films. ${ }^{157}$ More importantly, simultaneously enhancing the intrinsic conductivity and the active site exposure is crucial to boost the HER performance. Such dual advantage brought by structural and electronic modification was reported by Xie's group. ${ }^{158}$ They controllably incorporated oxygen in ultrathin $\mathrm{MoS}_{2}$ nanosheets and obtained a disordered structure whose unsaturated sulfur atoms flourished abundantly. Oxygen incorporation enlarged the interlayer spacing (Fig. 6f) and hence improved the conductivity. The HER performance with a current density of $126.5 \mathrm{~mA} \mathrm{~cm}{ }^{-2}$ at overpotential of $300 \mathrm{mV}$ (in $0.5 \mathrm{M} \mathrm{H}_{2} \mathrm{SO}_{4}$ ) exhibited by oxygen incorporated $\mathrm{MoS}_{2}$ is reported to be 60 times larger that of the bulk $\mathrm{MoS}_{2}$. Such significant enhancement is due to the more active sites, disordering, and conductivity, expanded interlayer spacing. ${ }^{158}$ The comparison of different $\mathrm{MoS}_{2}$ based materials is depicted in Fig. 6g.

The design of ternary structures, such as incorporating another chalcogen element, ${ }^{159-162}$ such as $\operatorname{MoS}_{2 x} \mathrm{Se}_{2(1-x)}$, inaugurates the possibility of tuning the composition. Interestingly, these ternary TMDs demonstrate better HER performance than the binary counterparts. From theoretical perspectives, the free energy of $\mathrm{H}$ adsorption is weaker $(80 \mathrm{meV})$ on sulfided Mo edges as compared to selenided Mo edges (-140 meV). Thus, controllably adjusting the $\mathrm{S} / \mathrm{Se}$ ratio to thermoneutral $\left(\Delta G_{\mathrm{H}} \approx 0\right)$ leads to more feasible edges for HER. ${ }^{161}$ In this respect, Gong et al. ${ }^{161}$ followed a solution method to synthesize $\operatorname{MoS}_{2(1-x)} \mathrm{Se}_{2 x}$ nanoflakes at low temperature and in high yields demonstrating better activity than either $\mathrm{MoS}_{2}$ or $\mathrm{MoSe}_{2}$. Similarly, doping of Co into $\mathrm{MoS}_{2}$ is proved to enhance the catalytic activity of $\mathrm{MoS}_{2}$ via decreasing the $\Delta G_{\mathrm{H}}$ from the S-edge side. ${ }^{142,163}$ The shape of $\mathrm{MoS}_{2}$ crystal changes from truncated triangle to hexagonal upon incorporation of $\mathrm{Co}$ thereby giving rise to Co-binding S-edges, which is more catalytically active. ${ }^{164}$ It is noteworthy that the development of Mo dichalcogenides based electrocatalysts has reached a fascinating stage by phase transformation of the electrocatalyst. ${ }^{68,156}$ Owing to its metallic nature (mentioned above), $1 \mathrm{~T}-\mathrm{MoS}_{2}$ nanosheet endows a facile electron kinetics, excellent electrical transport and proliferation of catalytic active sites. Consequently, as can be seen from Fig. $7 \mathrm{a}$ and $\mathrm{b}$, the HER performance of $1 \mathrm{~T} \mathrm{MoS}_{2}$ is better than that of the $2 \mathrm{H}$ phase. ${ }^{70}$ It has been suggested that the edge sites are not the main active sites for $1 \mathrm{~T}$ phase unlike the thermodynamically favored $2 \mathrm{H}$-phase. As proof of concept, partial oxidation of the edges was conducted to bear out the fact that the HER catalytic activity of $1 \mathrm{~T}-\mathrm{MoS}_{2}$ relies on the basal plane unlike that of $2 \mathrm{H}-\mathrm{MoS}_{2}$. As is evident from the schematic and the TEM images (Fig. 7c-e), the edges are highly disordered after oxidation, while the basal plane of the nanosheets remains unaffected. Accordingly, the 
a

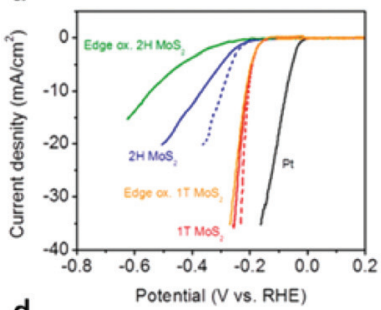

d

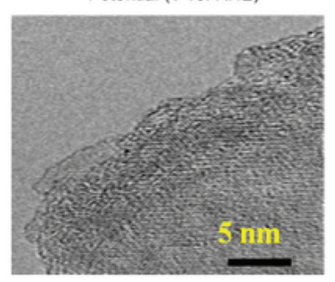

b

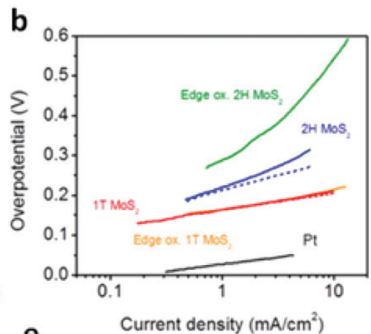

e

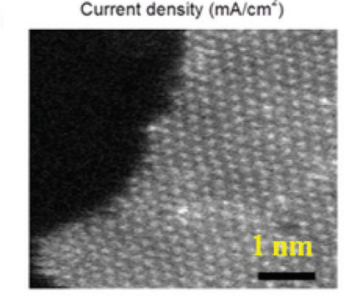

c
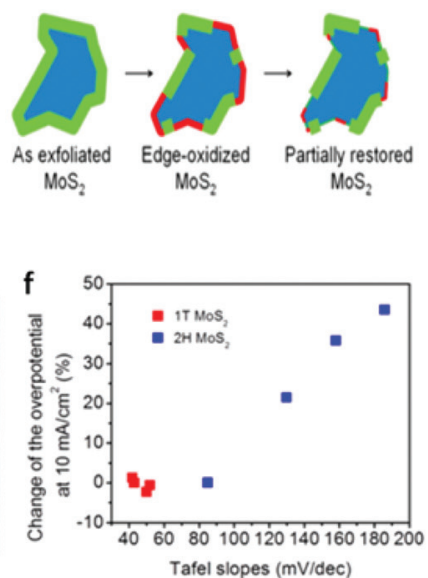

Fig. 7 (a) Polarization curves of $1 \mathrm{~T}$ and $2 \mathrm{H} \mathrm{MoS}_{2}$ nanosheet electrodes. (b) Corresponding Tafel plots. (c) Schematic of the oxidation process and partial restoration of the nanosheet edges after several voltammetric cycles. (d) High-resolution TEM (e) and HAADF STEMof edge-oxidized MoS 2 nanosheets showing corrugated edges caused by the chemical oxidization. (f) Variation of overpotential at $10 \mathrm{~mA} \mathrm{~cm}$ and Tafel slopes for $1 T$ and $2 \mathrm{H} \mathrm{MoS}_{2}$ (reproduced with permission from ref. 70. Copyright 2013 American Chemical Society).

electrochemical oxidation of the edges led to a dramatic reduction in catalytic activity for $2 \mathrm{H}$ nanosheets, but the catalytic performance was unaffected by oxidation for $1 \mathrm{~T}$ phase nanosheets. The overall performance of the oxidized $2 \mathrm{H}-\mathrm{MoS}_{2}$, however, improved after volumetric cycling in Fig. 7f, indicating that its catalytic activity from the edges was partially reestablished. These facts brought the conclusion that the basal plane is catalytically active for $1 \mathrm{~T}-\mathrm{MoS}_{2}$.

In general, the strategies employed so far for enhancing HER performance of molybdenum dichalcogenides based electrocatalysts include engineering of active edge sites or interlayer distances, hybridization with other conducting materials, addition of secondary metals or chalcogens, and the formation of $1 \mathrm{~T}$ phase.

3.1.1.2. Tungsten dichalcogenide based electrocatalysts. Owing to the structural resemblance between $\mathrm{WS}_{2}$ and $\mathrm{MoS}_{2},{ }^{163}$ those factors claimed to be detrimental for HER of molybdenum dichacogenides also apply for tungsten dichalcogenides and so the strategies set for achieving better performance. ${ }^{165}$ Thus, the development of tungsten dichalcogenide electrocatalysts is growing at comparable pace being a rival to that of molybdenum dichalcogenides. $\mathrm{WS}_{2}$, as an electrocatalyst for HER, was reported more than 25 years ago by Sobczynski et al. ${ }^{166}$ Different kinds of morphologies of $\mathrm{WS}_{2}$ including nanoflakes, ${ }^{167}$ nanoribbons, ${ }^{168}$ and nanosheets ${ }^{23}$ etc. have been introduced recently. Morphologies, such as nanotube (NT) and nanowire (NW), where the basal plane positioned parallel to the main axis are not required for HER purpose as this hides most of the active edges. ${ }^{169-171}$

Lin et al. ${ }^{168}$ unzipped $\mathrm{WS}_{2}$ NTs to fabricate a nanoribbon (NR) morphology that insures more exposed edge sites confirmed by significantly enhanced performance for NRs with smaller overpotential of $225 \mathrm{mV} v s$. RHE at $10 \mathrm{~mA} \mathrm{~cm} \mathrm{~cm}^{-2}$ as compared to that of NTs ( $420 \mathrm{mV} v s$. RHE). From the report by
Cheng et al., ${ }^{167}$ ultrathin nanoflakes of $\mathrm{WS}_{2}$ resulted in abundant accessible edges which ultimately contributed to HER performance of approximately $100 \mathrm{mV}$ of overpotential and

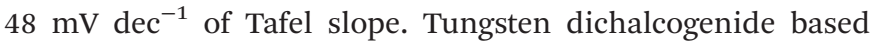
hybrid nanomaterials have also been reported to be HER active due to synergism. Yang et al. ${ }^{173}$ prepared $\mathrm{WS}_{2} / \mathrm{rGO}$ nanosheets in which the rapid electron transport gave rise to the record of overpotential ranging from 150 to $200 \mathrm{mV}$ with Tafel slope of $58 \mathrm{mV} \mathrm{dec}{ }^{-1}$. The compatible match of $\mathrm{WS}_{2}$ and rGO is very critical for establishing a pronounced synergism. Inspired by this, we recently ${ }^{174}$ reported self-supported and vertically aligned $\mathrm{WS}_{2}$ nanosheet on $\mathrm{W}$ foil sensitized by rGO for HER catalysis. The ultrathin rGO nanosheets on $\mathrm{WS}_{2}$ nanosheets contribute to the improvement of electron mobility. Significantly, the catalytic active edges of the vertical $\mathrm{WS}_{2}$ nanosheets have a direct contact with the electrolyte. These two factors efficiently improve the utilization rate of electrons, resulting in a decrease of the overpotential for HER. Meanwhile, the design of ternary dichalcogenides can realize the versatile change in their electronic structure and band gap. The recently reported results ${ }^{29,112,175}$ indicate the great potential for industry scale catalysts applications of the ternary tungsten dichalcogenides. In this respect, our group have controllably synthesized ternary $\mathrm{WS}_{2(1-x)} \mathrm{Se}_{2 x} \mathrm{NTs}^{29}$ from $\mathrm{WO}_{3}$ nanowire precursors on carbon fiber substrate (Fig. $4 \mathrm{a}-\mathrm{c}$ and $8 \mathrm{a}$ ). The sulfurization and selenization process led to dislocations and defects which is apparent from Fig. 8b, acting as active sites, on the nanotubes' walls. There, we clearly demonstrated how ternary TMDs are superior as HER catalysts as compared to the binary ones. Fig. 8c and d shows the result of HER measurement where it is evident that neither $\mathrm{WS}_{2}$ nor $\mathrm{WSe}_{2}$ NTs are as efficient as the ternary $\mathrm{WS}_{2(1-x)} \mathrm{Se}_{2 x}$ NTs. The low Tafel slopes recorded considerably evidence the fast HER rate at moderately increased overpotential. However, the confined number of the 

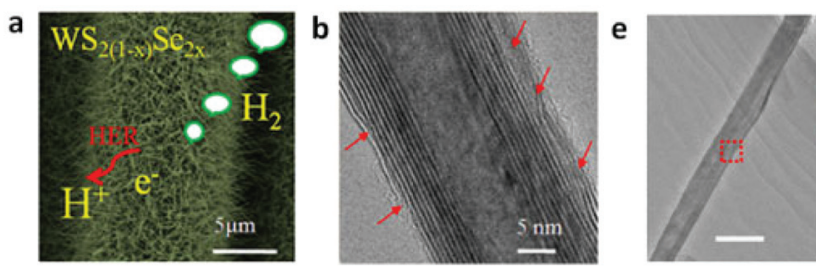

C
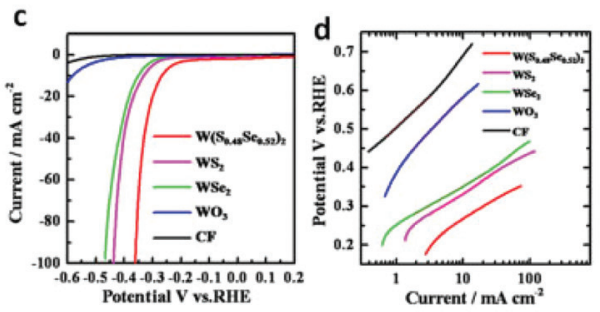

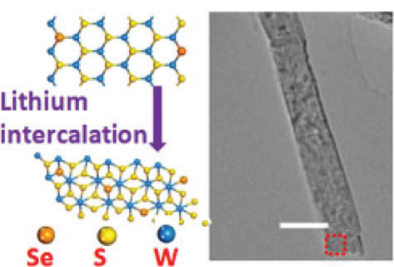

g

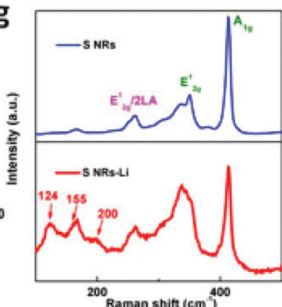

Fig. 8 (a) HRTEM image with many defects and dislocations indicated by red arrows in $\mathrm{WS}_{2(1-x)} \mathrm{Se}_{2 x} \mathrm{NT}$. (b) SEM image of ternary WS $\mathrm{S}_{2(1-x)} \mathrm{Se}_{2 x}(x=$ $0.52)$ NTs on CFs. (c) Polarization curves (after iR correction) of $\mathrm{WO}_{3} \mathrm{NWs}$ and $\mathrm{WS}_{2}, \mathrm{WSe}_{2}$ and $\mathrm{WS}_{2(1-x)} \mathrm{Se}_{2 x}(x=0.52) \mathrm{NTs}$ along with that corresponding to pure CFs for comparison. (d) Corresponding Tafel plots obtained from the polarization curves of pure CFs, WO $\mathrm{NWs}_{3}$ and WS $\mathrm{WSe}_{2}$ and $\mathrm{WS}_{2(1-x)} \mathrm{Se}_{2 x}(x=0.52) \mathrm{NTs}$ on CFs (reproduced with permission from ref. 29. Copyright 2014 American Chemical Society). (e) TEM images of $W_{2(1-x)} \mathrm{Se}_{2 x}$ NRs before and after lithium intercalation process (scale bar, $100 \mathrm{~nm}$ ). (f) AFM measurement of $\mathrm{WS}_{2(1-x)} \mathrm{Se}_{2 x} \mathrm{NRs}$ after lithium intercalation (scale bar, $200 \mathrm{~nm}$ ). (g) Raman spectra of $\mathrm{WS}_{2(1-x)} \mathrm{Se}_{2 x}$ NRs before and after lithium intercalation (reproduced with permission from ref. 172. Copyright 2015 John Wiley \& Sons, Inc.).

exposed edge sites on the NTs hinder the electrocatalytic activity of $\mathrm{WS}_{2(1-x)} \mathrm{Se}_{2 x}$. Following this result, we unzipped these NTs into nanoribbons (NRs) with $2 \mathrm{H}$ phase to increase the number of active sites. ${ }^{172}$ Likewise, the ternary system made through incorporation of another metal atom was also found to lead to substantial improvement in the catalytic activity. Catalytic dehydrogenation and hydrodesulfurization of organic compounds by TMDs ${ }^{176,177}$ were improved upon incorporation of VIII metal atoms ( $\mathrm{Ni}$ or $\mathrm{Co}$ ). Inspired by this, Sobczynski et al. ${ }^{178}$ studied the catalytic hydrogen evolution reaction of Ni-doped $\mathrm{WS}_{2}$. Nickel doping, forming mixed compounds (nickel and tungsten sulfide) on the catalyst surface, improved the catalytic hydrogen evolution properties of $\mathrm{WS}_{2}$. Also, the introduction of $\mathrm{Ni}$, Co or Mo into the $\mathrm{WS}_{2}{ }^{175}$ alters the chemical bonding states, thereby increasing the density of active sites on the surface. Even more impressively, the $\mathrm{WS}_{2}$ nanosheets with $1 \mathrm{~T}$ phase also facilitate hydrogen evolution with very low overpotentials. ${ }^{23}$ The high concentration of the strained metallic $1 \mathrm{~T}$ phase is associated with enhanced electrocatalytic ability. The DFT calculations show that the tensile strain on the basal plane can significantly influence the free energy of atomic hydrogen adsorption. ${ }^{23}$ After introducing a strain value of $2.7 \%$ on the surface, a free energy equal to zero can be extrapolated. Lukowski and coworkers ${ }^{63}$ reported that the $1 \mathrm{~T}-\mathrm{WS}_{2}$ nanosheets exfoliated after lithium intercalation showed dramatically improved HER with an overpotential of $142 \mathrm{mV}$ (vs. RHE) at $10 \mathrm{~mA} \mathrm{~cm}{ }^{-2}$. Recently, our group realized the phase conversion from $2 \mathrm{H}$ to $1 \mathrm{~T}$ phase of ultrathin ternary $\mathrm{WS}_{2(1-x)} \mathrm{Se}_{2 x}$ NRs by lithium intercalation reaction (Fig. 8e and f). ${ }^{172}$ As shown in Fig. 8g, the emergence of new Raman shifts at $\approx 124, \approx 155$ and $\approx 200 \mathrm{~cm}^{-1}$ at low frequency are associated with the phonon modes of $\mathrm{WS}_{2(1-x)} \mathrm{Se}_{2 x}$ contained in the $1 \mathrm{~T}$ phase, which achieves the lowest overpotential of $\approx 0.17 \mathrm{~V}$ at $10 \mathrm{~mA} \mathrm{~cm}{ }^{-2}$ and a Tafel slope of $\approx 68 \mathrm{mV} \mathrm{dec}^{-1}$.

3.1.1.3. Pyrite phase structured transition-metal dichalcogenide based electrocatalysts. The family of pyrite phase metal dichalcogenides ( $\mathrm{MX}_{2}$, where $\mathrm{M}=\mathrm{Fe}, \mathrm{Co}$, or $\mathrm{Ni}$ and $\mathrm{X}=\mathrm{S}$ or $\mathrm{Se}$ ) has been widely identified as potential electrocatalysts for HER in both acidic and alkaline conditions. They usually appear in cubic pyrite-type or orthorhombic macarsite-type structures in which the metal atoms are octahedrally bonded to adjacent chalcogen atoms (S and Se). The biomimetic speculation discussed above (Fig. 5a) also applies for this family due to the involvement of the metals in the metalloenzymes such as hydrogenase and nitrogenase. ${ }^{35}$ Theoreticians put forward that the free energy barrier for $\mathrm{H}$ adsorption on Co atom is low. ${ }^{134,179}$ Particularly, $\mathrm{CoS}_{2}{ }^{180}$ and $\mathrm{CoSe}_{2}{ }^{181}$ are intrinsically conductive and stable materials possessing an advantage over the commonly known $\mathrm{MoS}_{2}{ }^{68,70,156}$ and $\mathrm{WS}_{2}{ }^{63}$ as they exhibit remarkable HER activity without phase transformation requirement. Guided by this, various cobalt dichalcogenides materials including $\mathrm{CoS}_{2}$ nanostructures ${ }^{122,182,183}$ and $\mathrm{CoSe}_{2}$ nanostructures ${ }^{41,126,175,184}$ have been reported. The smallest ever reported Tafel slope $\left(\sim 30 \mathrm{mV} \mathrm{dec}{ }^{-1}\right)$ exhibited by $\mathrm{CoSe}_{2}$ mixed orthorhombic and cubic phases represents the best record, which is close to Pt, arising from the available surface that made the chemisorption of $\mathrm{H}$-atoms easier. ${ }^{184}$ Cobalt dichalcogenides based composites, such as $\mathrm{MoS}_{2} / \mathrm{CoSe}_{2}{ }^{91}$ and nickel/nickel(II) oxide nanoparticles/ $\operatorname{CoSe}_{2}{ }^{99}$ in which the electrocatalytic synergism effects are clearly demonstrated, have also been reported. For instance, Gao et al. grew $\mathrm{MoS}_{2}$ nanosheets on $\mathrm{CoSe}_{2}$ nanobelts to boost the HER performance in $0.5 \mathrm{M} \mathrm{H}_{2} \mathrm{SO}_{4}$ solution by utilization of synergetic chemical coupling effects (Fig. 9a-c). Fig. 9d reveals the fact that 

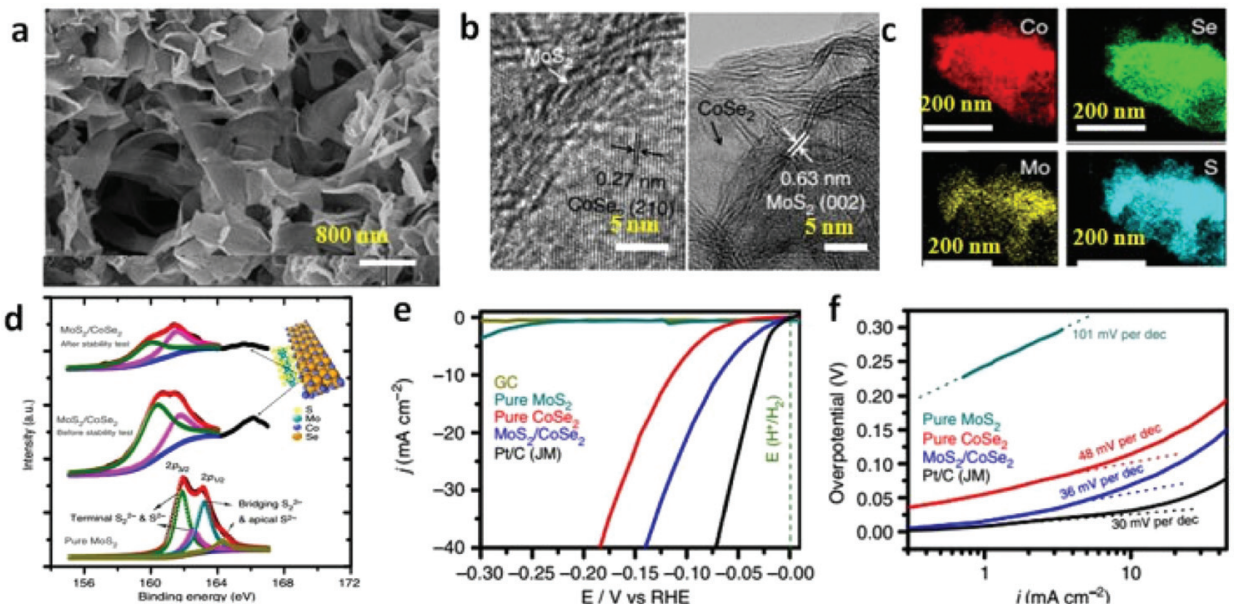

Fig. 9 (a) Scanning electron microscopy image of $\mathrm{MoS}_{2} / \mathrm{CoSe}_{2}$ hybrid. (b) HRTEM images of $\mathrm{MoS}_{2} / \mathrm{CoSe}_{2}$ hybrid showing distinguishable microstructures of $\mathrm{MoS}_{2}$ and $\mathrm{CoSe}_{2}$. (c) STEM-EDX elemental mapping of $\mathrm{MoS}_{2} / \mathrm{CoSe}_{2}$ hybrid showing clearly the homogeneous distribution of Co (red), Se (green), Mo (yellow) and S (azure). (d) S 2p XPS spectra for pure $\mathrm{MoS}_{2}, \mathrm{MoS}_{2} / \mathrm{CoSe}_{2}$ hybrid and $\mathrm{MoS}_{2} / \mathrm{CoSe}_{2}$ hybrid after stability test. The top right corner demonstrates the structure model of $\mathrm{MoS}_{2} / \mathrm{CoSe}_{2}$ hybrid. (e) Polarization curves for HER on different electrocatalysts. (f) Tafel plot for the various catalysts derived from (e) (reproduced with permission from ref. 87. Copyright 2015 Macmillan Publishers Limited).

chemical interaction between $\mathrm{CoSe}_{2}$ and $\mathrm{MoS}_{2}$ results in the formation of S-Co bonds, as observed from the generation of more terminal $\mathrm{S}_{2}{ }^{2-}$ and $\mathrm{S}^{2-}$ ions which are HER active. Thus, the $\mathrm{CoSe}_{2}$ nanobelts, assisted being a support for the growth of $\mathrm{MoS}_{2}$ with $\mathrm{S}_{2}{ }^{2-}$ and $\mathrm{S}^{2-}$ edges ultimately improve the HER performance. Their remarkable catalyst activity and fast HER kinetics were demonstrated as evidenced from the small onset potential and Tafel slope of $11 \mathrm{mV}$ (vs. RHE) and $36 \mathrm{mV} \mathrm{dec}^{-1}$, respectively (Fig. 9e and f). Comprehensively, D. Kong and coworkers ${ }^{40}$ identified the promising HER performances of firstrow transition-metal disulfides and diselenides $\left(\mathrm{FeS}_{2}, \mathrm{FeSe}_{2}\right.$, $\mathrm{FeCoS}_{2}, \mathrm{CoS}_{2}, \mathrm{CoSe}_{2}, \mathrm{CoNiSe}_{2}, \mathrm{CoNiS}_{2}, \mathrm{NiS}_{2}$ and $\mathrm{NiSe}_{2}$ ) prepared through sulfurization/selenization of e-beam-evaporated corresponding metal films. Collectively, their excellent HER catalysis performance from their thin films on glassy carbon in $0.5 \mathrm{M} \mathrm{H}_{2} \mathrm{SO}_{4}$ solution (Fig. 10) is revealed from a current density record of $4 \mathrm{~mA} \mathrm{~cm} \mathrm{~cm}^{-2}$ at potentials ranging from 190 to $270 \mathrm{mV}$. As evidenced from the summery of their Tafel slopes $\left(40-70 \mathrm{mV} \mathrm{dec}^{-1}\right)$, this family is characterized by relatively fast kinetics to drive HER and $\mathrm{CoS}_{2}, \mathrm{CoSe}_{2}$ and $\mathrm{NiS}_{2}$, possessed the smallest Tafel slope close to $40 \mathrm{mV} \mathrm{dec}{ }^{-1}$ relative to the others. Comparable Tafel slope is also reported for $\mathrm{NiS}_{2}$ nanosheeets. ${ }^{131}$ Among all the investigated materials, excellent HER performance was observed for $\mathrm{CoSe}_{2}$ and $\mathrm{FeCoS}_{2}$ due to their unique electronic structure. Notably, DFT calculation proved $^{185}$ that the kinetic energy barrier of $\mathrm{H}$ atom adsorption on $\mathrm{FeS}_{2}$ nanosheets was reduced upon doping of $\mathrm{Co}$, which ultimately resulted in higher HER activity. Following this observation, $\mathrm{CoSe}_{2}$ nanoparticles were prepared by templating commercial carbon black nanoparticles to expose more catalytic sites. ${ }^{41}$ The resulting $\mathrm{C} / \mathrm{CoSe}_{2}$ core shell significantly improved $\left(4 \mathrm{~mA} \mathrm{~cm}^{-2}\right.$ at $\left.90 \mathrm{mV}\right)$ the catalytic activity compared with $\mathrm{CoSe}_{2}$ film. This work was further extended by the same group in the report with an update of a more facile synthesis
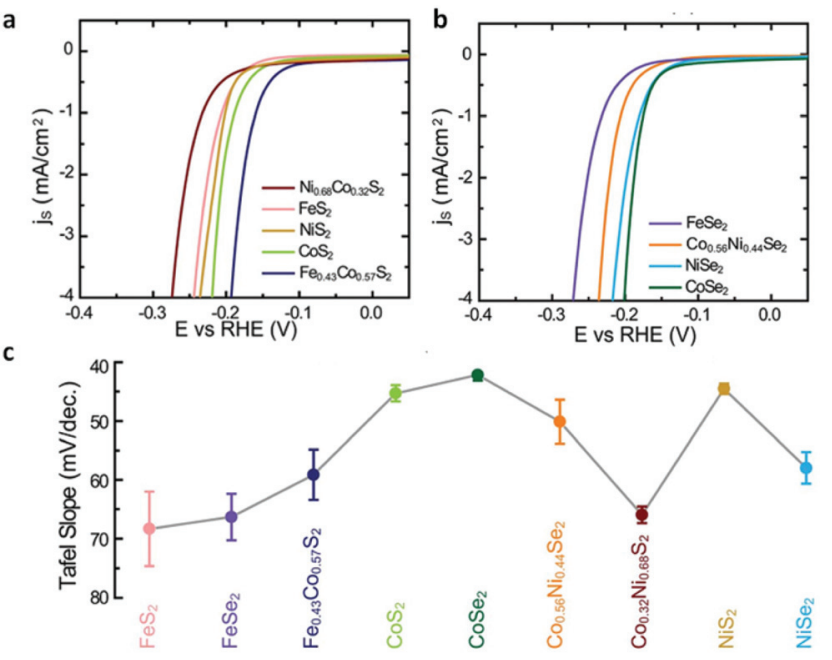

Fig. 10 (a) Polarization curves of transition-metal disulfides. (b) Polarization curves of transition-metal diselenides. (c) Summary of Tafel slopes of transition-metal dichalcogenide films (reproduced with permission from ref. 40. Copyright 2013 The Royal Society of Chemistry).

on 3D featured carbon fiber. Notably, substituting non-metal elements in these pyrite TMDs is an effective method to tune the hydrogen adsorption free energy. Recently, Jin's group ${ }^{186}$ found that the electrocatalytic property of pyrite $\mathrm{CoS}_{2}$ could be greatly improved by changing atomic components using phosphorus ions.

3.1.2. OER catalyzed by TMD based nanomaterials. As a half reaction involved in water splitting and containing complex electron transfer steps, an accumulation of the energy barrier in each step leads to the sluggish kinetics of OER with large overpotential $(\eta)$ to realize the oxygen evolution. 
Theoretically, the enthalpy of a lower to higher oxide transition, the $3 \mathrm{~d}$ electron number of bulk transition-metal ions, the surface oxygen binding energy and Yang Shao-Horn's principle, namely $\mathrm{e}_{\mathrm{g}}$ occupancy of the surface transition-metal cations is close to unity, are the OER activity descriptors. ${ }^{187}$ Among them, the Yang Shao-Horn's principle is a recently developed criterion to evaluate the OER activity deeply. Recently, an enormous number of cobalt based compounds, ${ }^{18}$ including simple metals, ${ }^{188-191}$ metal oxides ${ }^{192-196}$ hydro(oxy) oxides, ${ }^{197}$ phosphates, ${ }^{18,198-200}$ chalcogenides ${ }^{42,91}$ and perovskites $^{187,201}$ have been designed and utilized as electrocatalysts for oxygen evolution. ${ }^{44}$ Herein, we briefly review some recent results and the state-of-the-art in cobalt diachalcodenide based OER electrocatalysts, including one-component and hybrid ones, designed in the context of performance enhancement strategies.

According to the Yang Shao-Horn's principle, cubic cobalt dichalcogenide $\left(\mathrm{CoSe}_{2}\right)$ with a $\mathrm{t}_{2 \mathrm{~g}}{ }^{6} \mathrm{e}_{\mathrm{g}}{ }^{1}$ electronic configuration near the optimal $e_{g}$ filling (Fig. 11a and b) would be a terrific candidate for electrochemical water oxidation. ${ }^{43}$ Its large density of state (DOS) across the Fermi level by theoretical calculation demonstrates the metallic behavior (Fig. 11c). ${ }^{43}$ These intrinsic chemical and physical properties suggest and ensure efficient electron migration between the electrode and the surface of the catalyst for highly electrocatalytic OER performance. At the same time, the considerable active sites exposure yield also plays an important role on the superior OER performance. ${ }^{43}$ In order to further enhance the active site exposure, Liu et al. prepared atomically thick two-dimensional $\mathrm{CoSe}_{2}$ nanosheets, shown in Fig. 11d, through exfoliating the $\mathrm{CoSe}_{2}$-based inorganic-organic lamellar nanohybrids for electrocatalytic oxygen evolution. ${ }^{43}$ During the exfoliation process, a large number of Co vacancies $\left(V^{\prime \prime}{ }_{\mathrm{Co}}\right.$, Fig. $11 \mathrm{~d}$ and 12a), acting as active sites to catalyze the oxygen evolution reaction, formed. Compared with the bulk $\mathrm{CoSe}_{2}$ and the

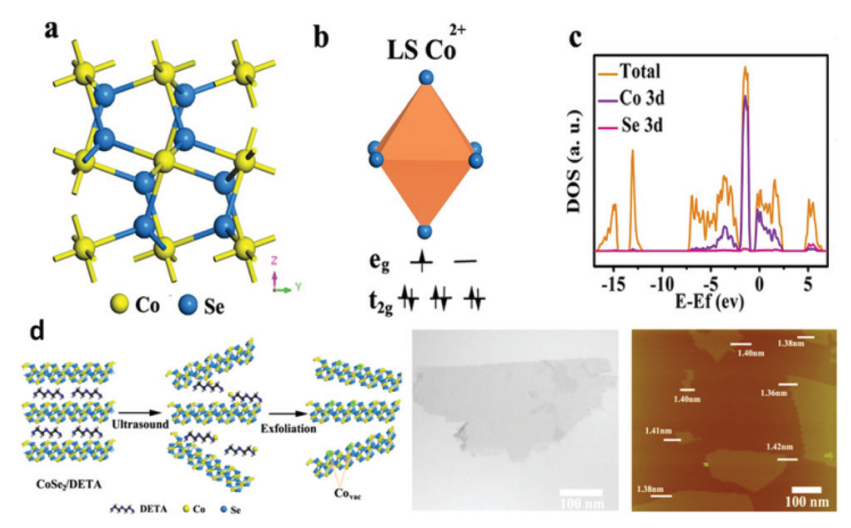

Fig. 11 (a) Crystal structure of $\mathrm{CoSe}_{2}$ in the cubic pyrite-type phase; (b) schematic spin structure of the Co cation; (c) DOS diagram for CoSe $\mathrm{C}_{2}$; (d) schematic of the formation of $V^{\prime \prime}{ }^{\prime}$ o vacancies in $\mathrm{CoSe}_{2}$ ultrathin nanosheets (left), TEM image (middle) and AFM image (right) of the ultrathin $\mathrm{CoSe}_{2}$ nanosheet (reproduced with permission from ref. 43. Copyright 2014 American Chemical Society).
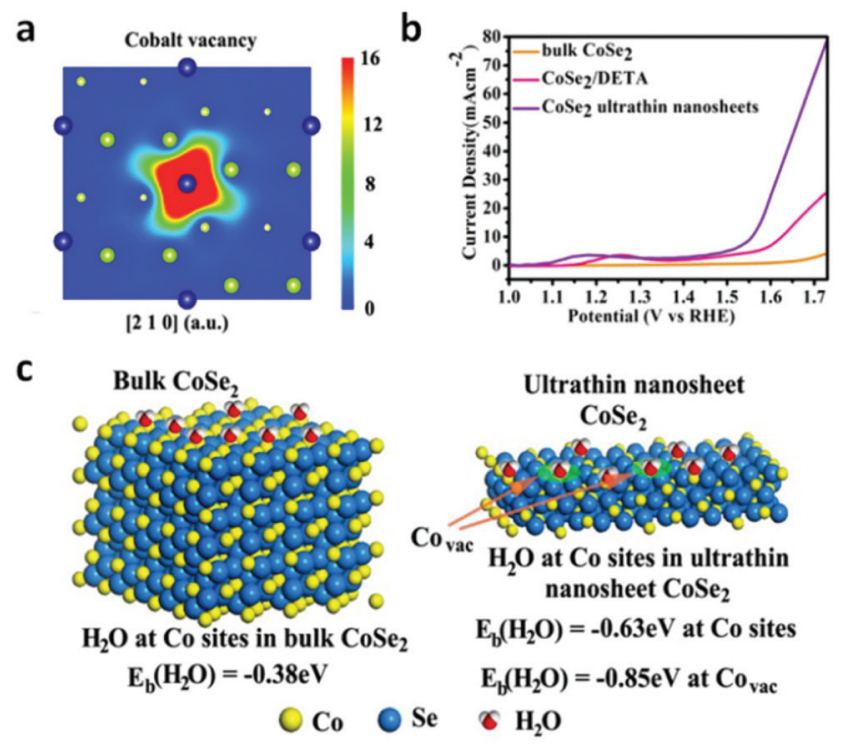

Fig. 12 (a) Schematic representations of trapped positrons of cobalt vacancies. (b) Linear sweep voltammetry (LSV) curves in $0.1 \mathrm{M} \mathrm{KOH}$ medium with bulk $\mathrm{CoSe}_{2}, \mathrm{CoSe}_{2} / \mathrm{DETA}$ and ultrathin $\mathrm{CoSe}_{2}$ nanosheet as the electrocatalyst. (c) First-principles study of surface $\mathrm{H}_{2} \mathrm{O}$ adsorption on different sites and performance of various materials: geometries and binding energies of $\mathrm{H}_{2} \mathrm{O}$ molecules on cobalt sites and vacancies (reproduced with permission from ref. 43. Copyright 2014 American Chemical Society).

nanohybrids, the ultrathin $\mathrm{CoSe}_{2}$ nanosheets exhibit the best OER performance (Fig. 12b) with small overpotential ( $0.32 \mathrm{~V}$ at $10 \mathrm{~mA} \mathrm{~cm}{ }^{-2}$ ) and Tafel slope (44 $\mathrm{mV} \mathrm{dec}^{-1}$ ) in basic solution (0.1 M KOH). Of particular note is that DFT calculated adsorption energy of $\mathrm{H}_{2} \mathrm{O}$ molecules on the atomically thick $\mathrm{CoSe}_{2}$ is much more favorable for adsorbing $\mathrm{H}_{2} \mathrm{O}$ molecules and facilitating the mass transport (Fig. 12c). This sample lost no activity after $1000 \mathrm{CV}$ cycles in $0.1 \mathrm{M} \mathrm{KOH}$, indicating the excellent stability of the ultrathin $\mathrm{CoSe}_{2}$ nanosheets.

To date, abundant studies demonstrate that the OER performance of Co-based electrocatalysts can be greatly improved through incorporation of other functional materials. ${ }^{99,202}$ In this regard, the synergetic chemical coupling effects between Co-based catalysts and foreign materials presumably lead to the substantial enhancement. Thus, additional modification of the $\mathrm{CoSe}_{2}$ with other materials, such as $\mathrm{Mn}_{3} \mathrm{O}_{4}$ nanoparticles, ${ }^{42}$ nitrogen-doped reduced graphene ${ }^{44}$ and $\mathrm{CeO}_{2}$ nanoparticles, ${ }^{45}$ were developed for boosting its OER performance by Yu's group. They successfully prepared ultrathin lamellar mesostructured $\mathrm{CoSe}_{2}$ /DETA nanobelts by solvothermal reaction. This mesostructure represents abundant and affordable material for large-scale utilization, large surface area and copious surface amino groups for anchoring the highly dispersed heteromaterials. ${ }^{42,98,202}$ As is shown in Fig. 13a-c, TEM images exhibit $\mathrm{Mn}_{3} \mathrm{O}_{4}$ nanoparticles with an average size of $\sim 15.7 \mathrm{~nm}$ uniformly distributed on the surface of the $\mathrm{CoSe}_{2}$ nanobelts. Bell et al. reported ${ }^{191}$ that Co cations in high oxidation states, namely $\mathrm{Co}^{\mathrm{IV}}$, have been proposed as active 

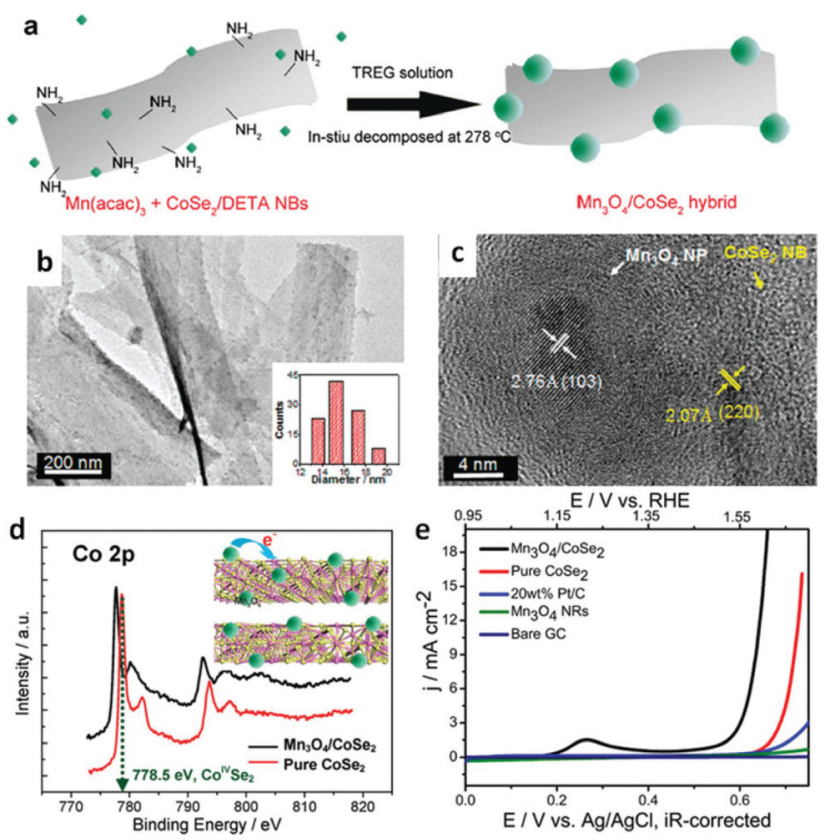

Fig. 13 (a) Polyol synthesis with $\mathrm{CoSe}_{2} / \mathrm{DETA}$ NBs to generate $\mathrm{Mn}_{3} \mathrm{O}_{4} /$ $\mathrm{CoSe}_{2}$ hybrid. (b) TEM images of $\mathrm{Mn}_{3} \mathrm{O}_{4} / \mathrm{CoSe}_{2}$ hybrid prepared at $278^{\circ} \mathrm{C}$ for $1 \mathrm{~h}$. The inset in (b) shows the corresponding particle-size histogram. (c) HRTEM image of an attached nanoparticle and its neighboring $\mathrm{CoSe}_{2}$ support. (d) Co $2 p$ XPS spectra for pure $\mathrm{CoSe}_{2} / \mathrm{DETA} \mathrm{NBs}$ and as constructed $\mathrm{Mn}_{3} \mathrm{O}_{4} / \mathrm{CoSe}_{2}$ hybrid. Inset demonstrates the electron donation from the $\mathrm{Mn}_{3} \mathrm{O}_{4}$ to $\mathrm{CoSe}_{2}$. Pink and yellow balls correspond to $\mathrm{Co}$ and Se atoms, respectively. (e) Polarization curves for OER on bare $\mathrm{GC}$ electrode and modified GC electrodes comprising the $\mathrm{Mn}_{3} \mathrm{O}_{4} \mathrm{NRs}$, 20 wt\% Pt/C, pure $\mathrm{CoSe}_{2} / \mathrm{DETA} \mathrm{NBs}$ and $\mathrm{Mn}_{3} \mathrm{O}_{4} / \mathrm{CoSe}_{2}$ hybrid, respectively (reproduced with permission from ref. 42. Copyright 2012 American Chemical Society). centers. The $\mathrm{Co}^{\mathrm{IV}}$ species existed in $\mathrm{CoSe}_{2}$ nanobelts were believed to enhance the electrophilicity of the adsorbed $\mathrm{O}$ and accelerate the formation of intermediate products, such as $\mathrm{O}-\mathrm{OH}$ and $\mathrm{OOH}$ species, to eventually generate $\mathrm{O}_{2}$ molecules. ${ }^{42}$ Fig. $13 \mathrm{~d}$ demonstrates the electron binding energy of Co 2p decreased after decorating the $\mathrm{CoSe}_{2}$ by $\mathrm{Mn}_{3} \mathrm{O}_{4}$ nanoparticles. This phenomenon is induced by the electron transfer from $\mathrm{Mn}_{3} \mathrm{O}_{4}$ to $\mathrm{CoSe}_{2}$, which makes the $\mathrm{Mn}_{3} \mathrm{O}_{4}$ more acidic (Lewis acid). Thus, the $\mathrm{Mn}_{3} \mathrm{O}_{4}$ nanoparticles anchored on the $\mathrm{CoSe}_{2}$ facilitates the activation of $\mathrm{H}_{2} \mathrm{O}$ (Lewis base) molecules through Lewis acid-base interaction. As a result, the $\mathrm{Mn}_{3} \mathrm{O}_{4} / \mathrm{CoSe}_{2}$ exhibited more improved OER electrocatalytic activity than others (Fig. 13e). Analogously, the $\mathrm{CeO}_{2}$ nanoparticles, creating much more oxygen vacancies, can sensitize the $\mathrm{CoSe}_{2}$ nanobelts to form hydroperoxy species $\left(\mathrm{OOH}_{\mathrm{ad}}\right)$ and further promote the OER catalytic activity. The $\mathrm{CeO}_{2} / \mathrm{CoSe}_{2}$ shows a small overpotential of $\sim 0.288 \mathrm{~V}$ at the current density of $10 \mathrm{~mA} \mathrm{~cm}{ }^{-2}$, small Tafel slope of $44 \mathrm{mV}$ $\mathrm{dec}^{-1}$, large anodic currents and good durability in alkaline solution. ${ }^{45}$ The chemical synergistic effect between $\mathrm{Mo}_{3} \mathrm{O}_{4}$ or $\mathrm{CeO}_{2}$ and $\mathrm{CoSe}_{2}$ guarantees increased OER activity relative to pure $\mathrm{CoSe}_{2}$.

Additionally, graphene sheets are becoming an inexpensively outstanding matrix to support electrocatalysts and facilitate catalytic performance through strong chemical coupling and optimized electronic structure. ${ }^{203,204}$ Fig. 14a and b shows that the long belt-like $\mathrm{CoSe}_{2}$ with size of $\sim 2-3 \mu \mathrm{m}$ and almost transparent features were immobilized on the $\mathrm{N}$-doped reduced graphene (NG) sheet $(>6 \mu \mathrm{m}){ }^{44}$ The thickness of the $\mathrm{CoSe}_{2}$ nanobelt and NG sheet is close to $\sim 19.6$ and $\sim 2.6 \mathrm{~nm}$, respectively (Fig. 14c). After the electrochemical measurement a

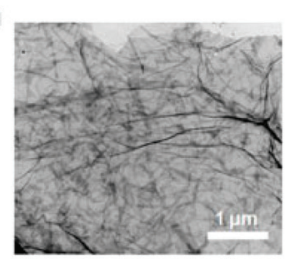

d

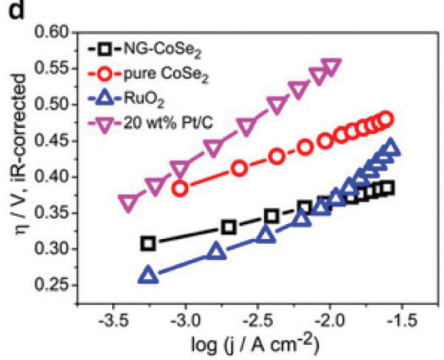

b
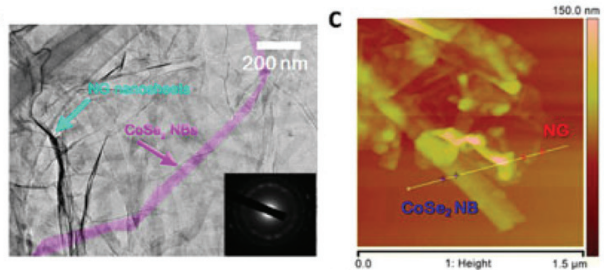

e $\quad \eta / \mathrm{V}$, iR-corrected

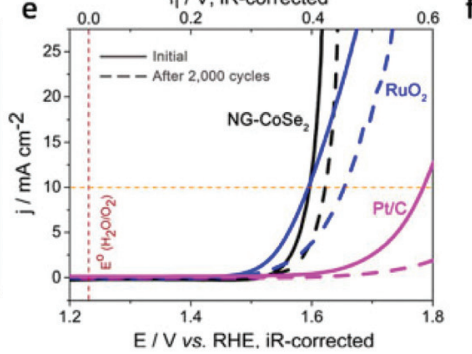

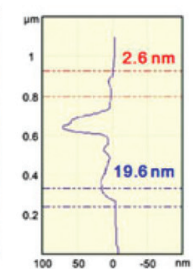

f

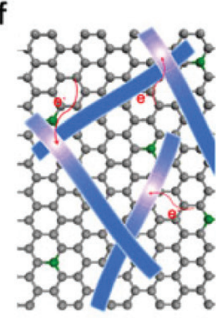

Fig. 14 (a-b) TEM images with different magnifications of NG-CoSe ${ }_{2}$ composite. The inset in (b) shows the corresponding SAED pattern. (c) AFM image and corresponding height profile of $\mathrm{NG}-\mathrm{CoSe}_{2}$ composite. (d-e) Tafel plot and polarization curves for OER on bare GC electrode and

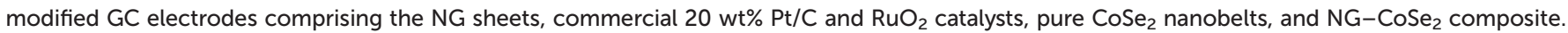
(f) Schematic image demonstrates the electron donation from the NG to $\mathrm{CoSe}_{2}$. Gray, green balls and blue belts correspond to $\mathrm{C}$, N atoms and $\mathrm{CoSe}_{2}$ nanobelts, respectively (reproduced with permission from ref. 44. Copyright 2014 American Chemical Society). 


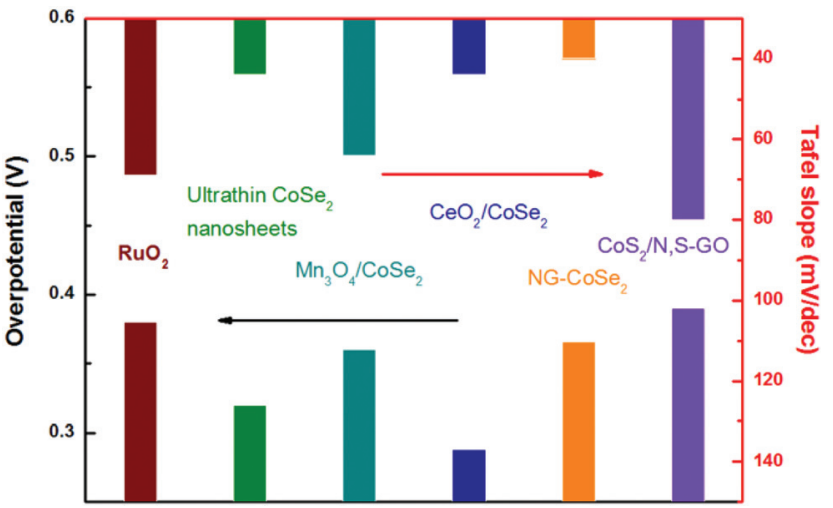

Fig. 15 OER performance comparison of different TMD based electrocatalysts with $\mathrm{RuO}_{2}$ (data gathered from ref. 42-45 and 206).

in the $0.1 \mathrm{M} \mathrm{KOH}$ electrolyte, the polarization curve of NG-CoSe ${ }_{2}$ composite shows a much earlier onset potential.

Moreover, the corresponding Tafel plots displayed in Fig. 14d exhibits the smallest Tafel slope $\left(40 \mathrm{mV} \mathrm{dec}^{-1}\right)$ for the hybrid sample, reveals that the composite outperforms other catalysts, including commercial $\mathrm{RuO}_{2}$, pure $\mathrm{CoSe}_{2}$ nanobelts, and commercial Pt/C. Importantly, the comparison of the original polarization curve and that of after 2000 cycles of NG$\mathrm{CoSe}_{2}$ and $\mathrm{RuO}_{2}$ demonstrates the superiority and stability of the material for OER property in basic media (Fig. 14e). In this system, the largely exposed $\mathrm{CoSe}_{2}$ surfaces, tightly bound by the NG sheet, were utilized as active substance. The introduction of the NG sheets can afford high electrical conductivity and prevent undesired aggregation. From the d-band theory, ${ }^{187,205}$ the interaction between oxygen and metal d states, including the bond strength of oxygen-related intermediate species, can be optimized via modulating the d-electron filling in $e_{g}$ bands. Therefore, the electron donation from NG sheets to $\mathrm{CoSe}_{2}$ afford more $\mathrm{e}_{\mathrm{g}}$-filling in $\mathrm{CoSe}_{2}$ (Fig. 14f), which could optimize the surface-oxygen interaction to moderate the bond strength, thereby greatly enhancing the OER kinetics. Meanwhile, nitrogen and sulfur co-doped graphene oxide also used to anchor the $\mathrm{CoS}_{2}$ nanoparticles for considerable OER performance. ${ }^{206}$ The electrocatalytic performance of different TMD-based electrocatalysts is summarized in Fig. 15, demonstrating the practically potential application although the real understanding of the contribution of cobalt dichalcogenide framework for the observed catalytic activity needs further investigation and confirmation. It is noteworthy that due to the likely chemical instability of common metal dichalcogenides in the oxidative and basic conditions, the stability of these $\mathrm{MX}_{2}$-based electrocatalysts for OER needs to be carefully studied and verified.

\subsection{Photochemical water splitting}

Photocatalytic water splitting, using solar energy to produce $\mathrm{H}_{2}$, is considered as a promising technology to address environmental crises and energy shortages. ${ }^{207-212}$ Solar water splitting based on inorganic semiconductors as a means of converting solar energy to chemical energy in the form of fuels has been drawn much attention since the 1970s. ${ }^{208,213}$ Typically, the photocatalytic ability of a semiconductor depends on the absolute positions of the valence band $\left(E_{\mathrm{VB}}\right)$ and conduction band $\left(E_{\mathrm{CB}}\right)$ edges. If its $E_{\mathrm{CB}}$ is located more negative than that of hydrogen $\left(E_{\mathrm{H}_{2} / \mathrm{H}^{+}}\right)$and the $E_{\mathrm{VB}}$ is more positive than that of oxygen $\left(E_{\mathrm{O}_{2} / \mathrm{H}_{2} \mathrm{O}}\right)$, it is possible to split the water molecule into $\mathrm{H}_{2}$ and $\mathrm{O}_{2}$ under light. A proper band gap is therefore of primary importance for photocatalytic reactions. ${ }^{208}$ Furthermore, the composite photocatalysts, including hybrids with other semiconductors, carbon materials and noble metals, are extensively studied due to improved carrier diffusion and efficient absorption of the solar spectrum., ${ }^{8,214}$ TMDs, especially Mo and $\mathrm{W}$ based ones, have been used as photoelectrodes for water splitting due to the following advantages: ${ }^{215,216}$ (i) their band gaps ranges from 1.0 to $1.6 \mathrm{eV}$, which are well matched with the solar spectrum. (ii) These semiconductors should be quite stable towards to photodissolution because of $\mathrm{d}-\mathrm{d}$ optical transitions and involving nonbonding electrons. For example, the stability of the $\mathrm{MoS}_{2}$ photoelectrode has been associated with the fact that the electronic excitation does not involve a transition having $\mathrm{S}^{2-} \rightarrow \mathrm{Mo}(\mathrm{Iv})$ charge-transfer character. (iii) They are earth-abundant and composed of non-precious materials. However, TMD nanostructures are usually utilized as cocatalysts for photocatalytic $\mathrm{H}_{2}$ generation, presently, because their conduction band position is inadaptable to reduce $\mathrm{H}^{+}$to $\mathrm{H}_{2}$ under light illumination. In this section, we summarize the photocatalytic properties of both single component and composite TMD based on very commonly used $\mathrm{MoS}_{2}$ and $\mathrm{WS}_{2}$ materials. In particular, monolayer $\mathrm{MoS}_{2}$ and the heterostructures, in which $\mathrm{MoS}_{2}$ and $\mathrm{WS}_{2}$ act as cocatalysts, are highlighted.

3.2.1. Monolayer $\operatorname{MoS}_{2}$ and its photocatalytic properties. The optical absorption spectrum of $\mathrm{MoS}_{2}$ nanomaterials has been calculated and thoroughly studied experimentally. ${ }^{32,218,219}$ DFT calculations and experimental results show that the band gap of $\mathrm{MoS}_{2}$ would be increased dramatically when the sample thickness is reduced from a few layers to an ideal monolayer. ${ }^{32,220,221}$ The direct gap of monolayer $\mathrm{MoS}_{2}$ is about 1.9 $\mathrm{eV}$, which is larger than that of bulk $\mathrm{MoS}_{2}(1.29 \mathrm{eV}) .^{32,222,223}$ Notably, the increased band gap of the $\mathrm{MoS}_{2}$ leads to a change in the redox potential and improves the photocatalytic property. Fig. 16 presents the energy diagrams of the $\mathrm{VB}$ and $\mathrm{CB}$ edge potentials in bulk and monolayer $\operatorname{MoS}_{2} \cdot{ }^{217}$ It is found that the bulk $\mathrm{MoS}_{2}$ can oxidize $\mathrm{H}_{2} \mathrm{O}$ to produce $\mathrm{O}_{2}$ but can not reduce $\mathrm{H}^{+}$to $\mathrm{H}_{2}$. In contrast, the monolayer $\mathrm{MoS}_{2}$ can oxidize $\mathrm{H}_{2} \mathrm{O}$ more efficiently than bulk $\mathrm{MoS}_{2}$. The CB edge position of the monolayer $\mathrm{MoS}_{2}$ is more negative than that of $\mathrm{H}^{+} / \mathrm{H}_{2}$, suggesting its photocatalytic potential to reduce $\mathrm{H}^{+}$for $\mathrm{H}_{2}$. However, the research on the direct use of $\mathrm{MoS}_{2}$ as photocatalyst is rarely reported due to the low-yield production of monolayer $\mathrm{MoS}_{2}$ nanosheets. ${ }^{224}$

3.2.2. TMDs as cocatalysts for photocatalytic water splitting. As a thermodynamically uphill reaction, water splitting is a non-spontaneous process. Thus, some cocatalysts usually are 


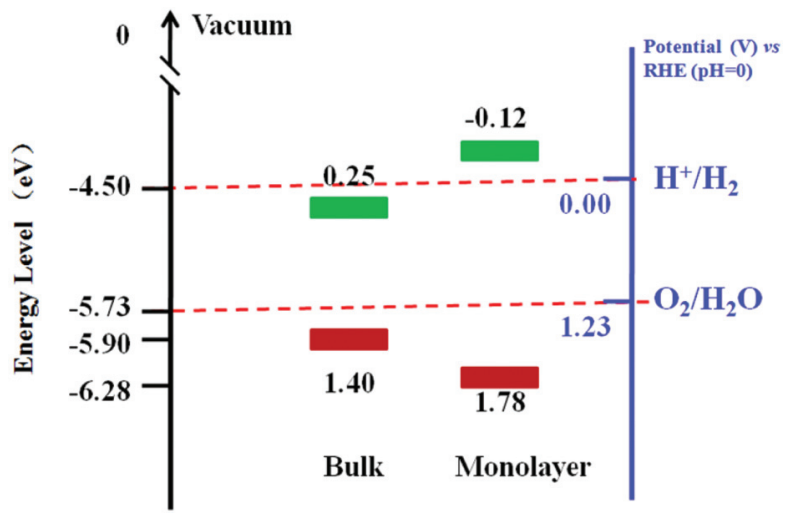

Fig. 16 Energy diagrams of the $V B$ and $C B$ edge potentials in bulk and monolayer $\mathrm{MOS}_{2}$ (data gathered from ref. 217).

loaded on the semiconductors that act as photo-harvesters to reduce the energy barrier (Scheme 1). At the same time, the cocatalyst is capable of assisting in photo-generated electronhole separation and suppressing the photo-corrosion to increase the stability of semiconductor photocatalysts. ${ }^{49}$ To date, many TMDs, i.e. $\mathrm{MoS}_{2},{ }^{50-52,225-227} \mathrm{WS}_{2}{ }^{53,214}$ and $\mathrm{NiS}_{2},{ }^{228}$ have been reported as excellent candidates for cost-effective cocatalysts.

Due to its high mobility for charge transport, $\mathrm{MoS}_{2}$ nanostructure as a cocatalyst can enhance the separation of photoexcited electrons and holes as well as decrease the activation potentials for $\mathrm{H}_{2}$ evolution, which is paramount for developing photocatalytic water splitting activity of photocatalysts. For instance, $\mathrm{TiO}_{2}$, as a typical UV light driven photocatalyst, has been reported to show enhanced photocatalytic $\mathrm{H}_{2}$ production activity by loading $\mathrm{MoS}_{2}$ nanostructures. ${ }^{225,230-233}$ In order to improve interfacial charge transfer, Xiang's group ${ }^{226}$ reported

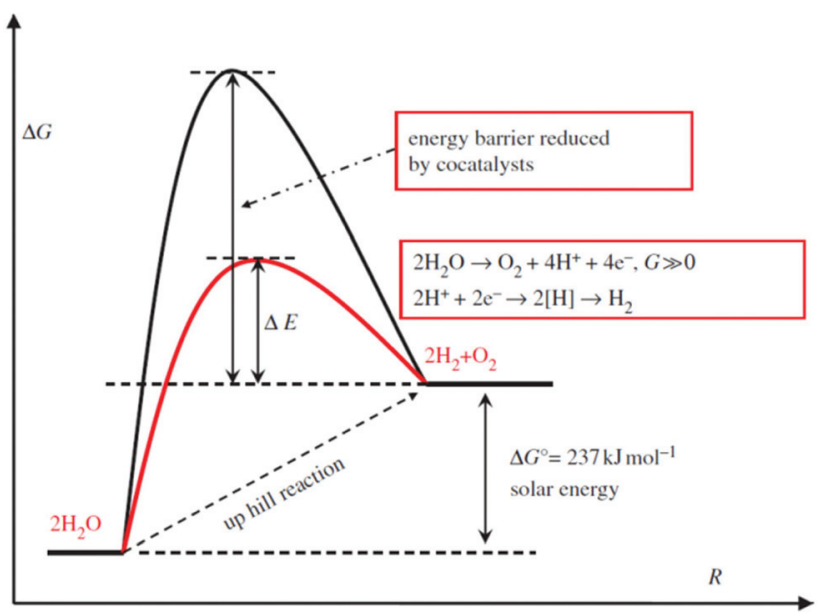

Scheme 1 Schematic description of energy diagram for photocatalytic water splitting (reproduced with permission from ref. 212. Copyright 2013 Royal Society). a $\mathrm{TiO}_{2} / \mathrm{MoS}_{2} /$ graphene composite demonstrating a 7-times $\mathrm{H}_{2}$ production rate improvement than that obtained from $\mathrm{TiO}_{2}$ alone under UV irradiation. Besides, as is known to us, single component visible light driven photocatalysts face the limitation of photoelectric conversion efficiency and low photocatalytic activity. ${ }^{8,234-236}$ Nowadays, therefore various visible light driven photocatalysts, such as $\mathrm{CdS},{ }^{237} \mathrm{Zn}_{0.2} \mathrm{Cd}_{0.8} \mathrm{~S},{ }^{238}$ $\mathrm{CdSe}^{227}$ have been studied in combination with TMDs to obtain higher photocatalytic efficiency. Zong et al. ${ }^{237}$ reported enhancement of the photocatalytic $\mathrm{H}_{2}$ production activity of an $\mathrm{MoS}_{2}-\mathrm{CdS}$ compound. Subsequently, Zhang's group ${ }^{229}$ employed a facile one-pot wet-chemical method to fabricate $\mathrm{MoS}_{2}-\mathrm{CdS}$ and $\mathrm{WS}_{2}-\mathrm{CdS}$ nanohybrids. They found that the single-layered $\mathrm{MoS}_{2} / \mathrm{WS}_{2}$ selectively grew on the Cd-rich (0001) surface of wurtzite CdS nanocrystals, wherein the strong interaction with $\mathrm{S}$ layer of $\mathrm{MoS}_{2} / \mathrm{WS}_{2}$ occurred. This kind of nanohybrids reduces charge carrier recombination and photocorrosion of CdS, leading to excellent photocatalytic activity with good stability. As shown in Fig. 17, they achieved a hydrogen evolution rate of $1472 \mu \mathrm{mol} \mathrm{h}^{-1} \mathrm{~g}^{-1}$ with $\mathrm{MoS}_{2}$-CdS nanohybrids, which is 12 times higher than that of pure CdS nanocrystals, and this photocatalyst still remained catalytically active in $16 \mathrm{~h}$ irradiation under visible light. To get better photocatalytic $\mathrm{H}_{2}$ production activity, Yang et al..$^{51}$ introduced metal nanoparticles ( $\mathrm{Cr}, \mathrm{Ag}$ ) to the surface of $\mathrm{MoS}_{2}$ nanosheets by a simple solution method and then loaded onto a CdS catalyst. With this metal-MoS${ }_{2}$ heterostructure, the recombination of photogenerated carriers in $\mathrm{MoS}_{2}$ nanosheets was reduced, leading to a great enhancement of photoelectric conversion efficiency. As a result, they attained an average rate of $38000 \mu \mathrm{mol} \quad \mathrm{h}^{-1} \quad \mathrm{~g}^{-1} \quad \mathrm{H}_{2}$ evolution under visible light
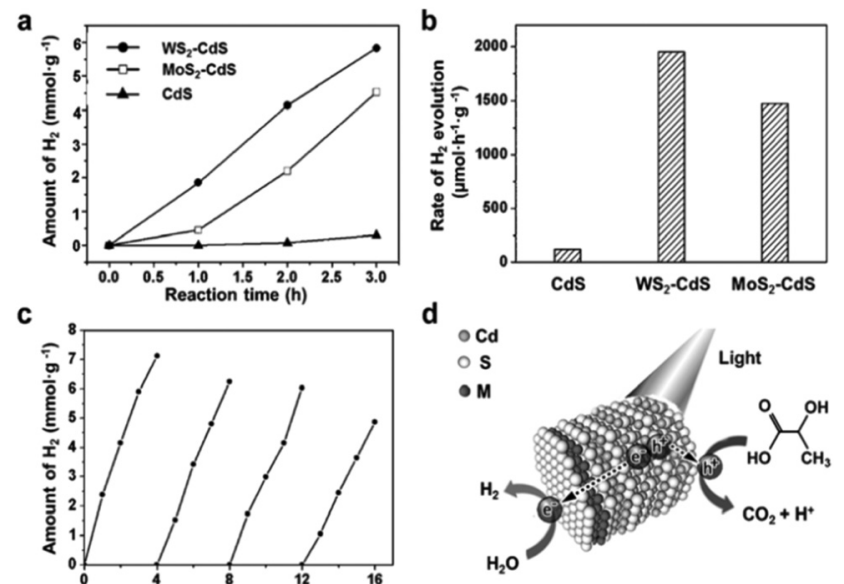

Fig. 17 Photocatalytic activity of $\mathrm{MS}_{2}-\mathrm{CdS}$ nanohybrids for hydrogen evolution reaction. (a) Time-dependent photocatalytic $\mathrm{H}_{2}$ evolution for $\mathrm{WS}_{2}-\mathrm{CdS}, \mathrm{MoS}_{2}-\mathrm{CdS}$ and pure CdS; (b) comparison of the $\mathrm{H}_{2}$ evolution rate under visible light irritation for $\mathrm{WS}_{2}-\mathrm{CdS}, \mathrm{MoS}_{2}-\mathrm{CdS}$ and pure CdS; (c) cycling test of photocatalytic $\mathrm{H}_{2}$ evolution for $\mathrm{WS}_{2}-\mathrm{CdS}$; (d) schematic illustration of the photocatalytic process of $\mathrm{MS}_{2}-\mathrm{CdS}$ nanohybrids in lactic acid solution (reproduced with permission from ref. 229. Copyright 2015 John Wiley \& Sons, Inc.). 
irradiation with $\mathrm{Cr}-\mathrm{MoS}_{2}$ hybrid as the cocatalyst. ${ }^{51}$ Furthermore, Chang and coworkers composited $\mathrm{MoS}_{2}$ nanosheets with graphene to anchor on CdS nanocrystals and achieved a $1.8 \mathrm{mmol} \mathrm{h}^{-1} \mathrm{H}_{2}$ evolution rate, which is much higher than that of CdS combined with noble metal Pt, in lactic acid solution under visible light. ${ }^{50}$ As environmental friendly and noble metal free photocatalysts, some carbon based materials have also been used for water splitting with $\mathrm{MoS}_{2}$ as cocatalyst. ${ }^{52,239}$ For example, Hou et al. ${ }^{52}$ fabricated graphitic carbon nitride and $\mathrm{MoS}_{2}$ layered nanojunctions to catalyze water for hydrogen evolution under visible light. They found that the $0.5 \mathrm{wt} \%$ $\mathrm{MoS}_{2} / \mathrm{mpg}-\mathrm{CN}$ (mesoporous g-CN) performs better than $0.5 \mathrm{wt} \% \mathrm{Pt} / \mathrm{mpg}-\mathrm{CN}$ with visible light irradiation. These results obviously suggest that $\mathrm{MoS}_{2}$ can be utilized to improve the poor hydrogen evolution kinetics of mpg-CN nanomaterial. Similar to $\mathrm{MoS}_{2}$, other TMDs such as $\mathrm{WS}_{2}$ and $\mathrm{NiS}_{2}$ also display a crucial role as cocatalyst in photocatalytic water splitting. Zong et al. ${ }^{53}$ reported that the $\mathrm{H}_{2}$ generation rate of CdS is increased by up to 28 times with $\mathrm{WS}_{2}$ as a good cocatalyst under visible light and also exhibits a persistently high $\mathrm{H}_{2}$-production capability in lactic acid solution. As such, ternary $\mathrm{CdLa}_{2} \mathrm{~S}_{4}$ with $2 \mathrm{wt} \% \mathrm{NiS}_{2}$ loading was also reported by Xue's group $^{228}$ as exhibiting a $\mathrm{H}_{2}$ production rate of $2.5 \mathrm{mmol} \mathrm{h}^{-1}$ $\mathrm{g}^{-1}$, which was 3 times higher than that of the pristine $\mathrm{CdLa}_{2} \mathrm{~S}_{4}$ photocatalyst. This was attributed to the enhanced separation of photogenerated electrons and holes as well as the activation effect of $\mathrm{NiS}_{2}$. The performance comparison of different photocatalysts with TMDs as cocatalyst for water splitting reported hitherto is demonstrated in Table 3. Moreover, owing to the feature of excellent electrocatalytic activity of layered TMDs, they are also utilized in the photoelectrochemical system for hydrogen generation. For example, $\mathrm{MoS}_{2}$ was employed as an electrocatalyst in the photocathode coupled by silicon nanowires. ${ }^{240,241}$ The dramatically enhanced current density along with significant anodic shift of onset potential suggested the highly active photoelectrochemical property for HER after preparation of the hybrid. Further, metallic $\mathrm{MoS}_{2}$ was integrated with p-Si to act as photocathode electrode, revealing high photocurrent density of $17.6 \mathrm{~mA} \mathrm{~cm}^{-2}$ at $0 \mathrm{~V}$ vs. RHE. $^{242}$

\subsection{Surface plasmon resonance (SPR) enhanced water splitting}

Surface plasmon resonance (SPR) results from the collective oscillation of the conduction electrons of plasmonic-metal, i.e. $\mathrm{Au}, \mathrm{Ag}$ and $\mathrm{Cu}$, nanoparticles. These surface plasmons predominantly relax non-radiatively via Landau damping, yielding highly energetic "hot" electrons emerging on the surface. ${ }^{243,244}$ When plasmonic-metal nanoparticles are proximal to a semihappen and boost the photocatalytic performance. ${ }^{244-246}$ Fang's group ${ }^{247}$ has reported that the plasmon-excited "hot" electrons can be injected into the $\mathrm{MoS}_{2}$ layer due to the low Schottky barrier between $\mathrm{MoS}_{2}$ and Au metal. In this situation, the $\mathrm{MoS}_{2}$ monolayers become acceptors of the "hot" electrons. Very recently, Shi et al. ${ }^{248}$ confirmed that these hot electrons conductor, a plasmon-mediated electron transfer process will

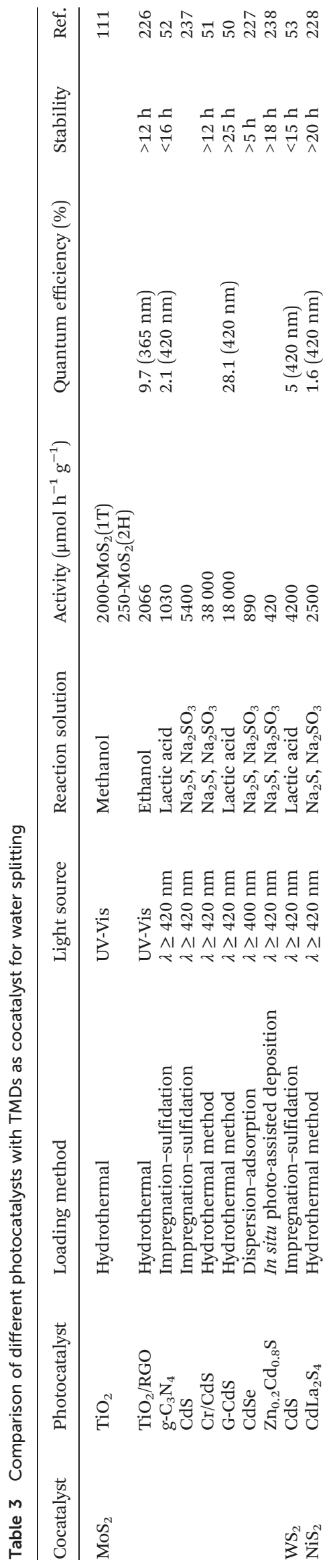


transferred from Au nanorod are suitable to facilitate the HER performance in the acid solution of $\mathrm{MoS}_{2}$ nanosheets (Fig. 18a and d). The overpotential and Tafel slope shown in Fig. 18b are reduced with $808 \mathrm{~nm}$ laser irradiation. The $i-t$ curves (Fig. 18c) indicate the good reversibility of this process. It is worth pointing out that the phase conversion from $2 \mathrm{H}$ to $1 \mathrm{~T}$ induced by SPR also plays a crucial role in promoting the electrocatalytic property. ${ }^{249}$ Yin et $a .^{250}$ found that the generated local electric field around $\mathrm{Au}$ nanoparticles promotes the photogenerated electron-hole separation in the $\mathrm{MoS}_{2}$ nanosheets. This phenomenon was suggested to enhance the photocatalytic water splitting in the photoelectrochemical cell using $\mathrm{Au}-\mathrm{MoS}_{2}$ composite.
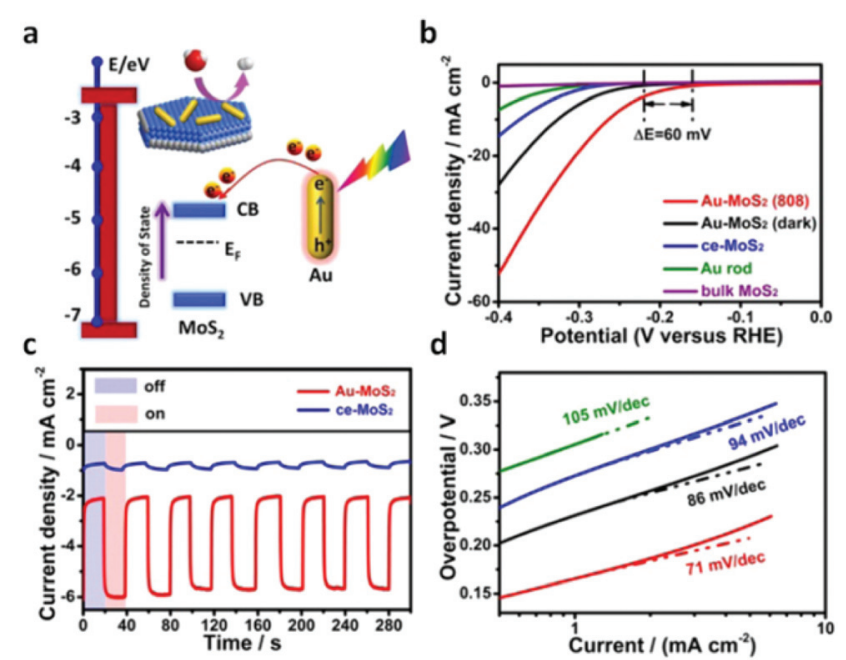

Fig. 18 (a) Schematic $\mathrm{Au}-\mathrm{MoS}_{2}$ and energy level diagram illuminating hot electrons injection and change of $\mathrm{MoS}_{2}$ Fermi level. (b) HER polarization curves obtained on several catalysts as indicated. (c) $i-t$ curves with and without $808 \mathrm{~nm}$ laser excitation. (d) The Tafel plots of $\mathrm{Au}$ rod (green), ce-MoS 2 (blue), Au-MoS 2 (black) and $\mathrm{Au}-\mathrm{MoS}$ (808) (red) derived from the early stages of HER polarization curves (reproduced with permission from ref. 248. Copyright 2015 American Chemical Society).

\section{Conclusion and perspectives}

Capturing energy from the sun and/or water provides a greener approach to emerge from the damaging fossil fuel era. Being emerging candidates for water splitting, TMD based nanomaterials are extensively studied as electrocatalysts and photocatalysts for hydrogen and oxygen production. This review provides a survey of literatures for the preparation and applications of various TMD materials in electrocatalytic and photocatalytic water splitting. Methods such as chemical exfoliation, solvothermal and chemical vapor deposition have been utilized, with modifications thereof, encompassing top-down and bottom-up approaches to synthesize high quality TMDs intended for water splitting. The morphology through which maximum active sites are exposed, synergism from the individual components in the hybrid structure and a $1 \mathrm{~T}$ phase of $\mathrm{MoS}_{2}$ and $\mathrm{WS}_{2}$ were directives reported so far for enhanced electrocatalytic HER or OER. More importantly, it is imperative to further consider Co-based dichalcogenides as potential materials in both HER and OER. Due to their unique electronic configuration near the optimal $\mathrm{e}_{\mathrm{g}}$ filling, electrodes built on the basis of Co dichalcogenides demonstrate excellent performance without the need of phase transfer. As one of the prospects, successful incorporation of heteroatoms into the lattice of other TMDs is speculated to greatly improve the electrocatalytic water splitting performance via modulating the electronic structure. Of particular note is that the monolayered TMDs are potential candidates for photocatalytic water splitting under visible light (Fig. 19), rendering the opportunity to harvest solar light for photocatalytic hydrogen or oxygen evolution (except for some metallic ones: labeled in red). Additionally, the SPR effect is also effectively employed to boost electroor photo-catalytic water splitting performance. Some other hybrid nanostructures composited with carbon materials, semiconductors and metals have also exhibited great promise for overcoming some of the limitations of TMDs.

A future direction, therefore, also lies in the design of novel TMD based nanomaterials aimed to exploit their new functionalities for efficient water splitting.
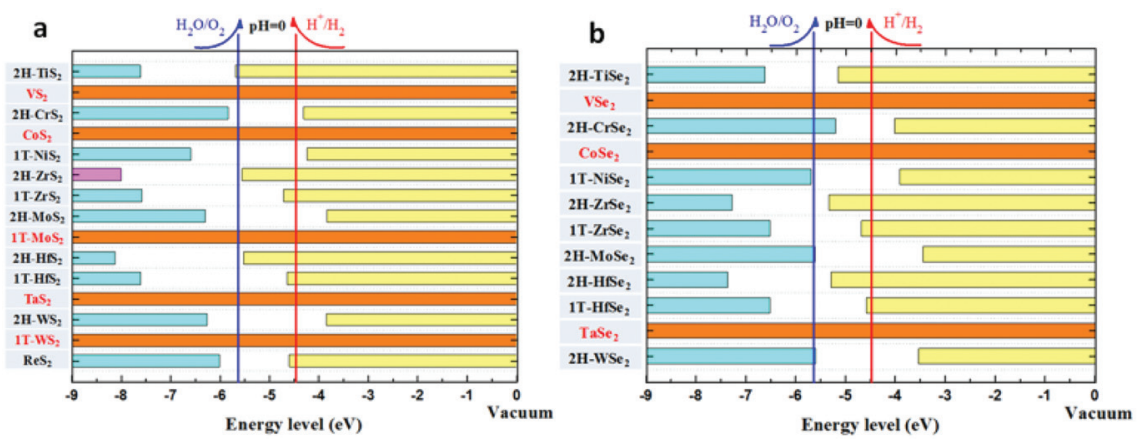

Fig. 19 Band edge positions of monolayer (a) sulfide and (b) selenide TMDs relative to the vacuum level, including the redox potentials for $\mathrm{H}^{+} / \mathrm{H}_{2}$ and $\mathrm{O}_{2} / \mathrm{H}_{2} \mathrm{O}$ at $\mathrm{pH}=0$. The band edge data are compiled from ref. 48 and 251-254. 


\section{Acknowledgements}

This work was supported by the National Natural Science Foundation of China (No. 21373065 and 61474033), 973 Program of the Ministry of Science and Technology of China (No. 2012CB934103), Beijing Natural Science Foundation (No. 2144059) and CAS Key Laboratory of Nanosystem and Hierarchical Fabrication. The authors gratefully acknowledge the support of K. C. Wong Education Foundation.

\section{Notes and references}

1 T. Hisatomi, J. Kubota and K. Domen, Chem. Soc. Rev., 2014, 43, 7520-7535.

2 M. R. Gholipour, C. T. Dinh, F. Béland and T. O. Do, Nanoscale, 2015, 7, 8187-8208.

3 J. M. Bockris, Int. J. Hydrogen Energy, 2002, 27, 731740.

4 J. R. Rostrup-Nielsen, Catal. Rev. Sci. Eng., 2004, 46, 247270.

5 J. Greeley, T. F. Jaramillo, J. Bonde, I. Chorkendorff and J. K. Norskov, Nat. Mater., 2006, 5, 909-913.

6 M. Chhowalla, H. S. Shin, G. Eda, L.-J. Li, K. P. Loh and H. Zhang, Nat. Chem., 2013, 5, 263-275.

7 C. Wang, Q. Hu, J. Huang, C. Zhu, Z. Deng, H. Shi, L. Wu, Z. Liu and Y. Cao, Appl. Surf. Sci., 2014, 292, 161-164.

8 Y. Wang, Q. Wang, X. Zhan, F. Wang, M. Safdar and J. He, Nanoscale, 2013, 5, 8326-8339.

9 T. Sun, E. Liu, X. Liang, X. Hu and J. Fan, Appl. Surf. Sci., 2015, 347, 696-705.

10 X. Fan, J. Wan, E. Liu, L. Sun, Y. Hu, H. Li, X. Hu and J. Fan, Ceram. Int., 2015, 41, 5107-5116.

11 M.-S. Park and M. Kang, Mater. Lett., 2008, 62, 183187.

12 C. G. Morales-Guio, L.-A. Stern and X. Hu, Chem. Soc. Rev., 2014, 43, 6555-6569.

13 M. Grätzel, Nature, 2001, 414, 338-344.

14 Y. Sun, S. Gao, F. Lei and Y. Xie, Chem. Soc. Rev., 2015, 44, 623-636.

15 Y. Sun, Z. Sun, S. Gao, H. Cheng, Q. Liu, J. Piao, T. Yao, C. Wu, S. Hu and S. Wei, Nat. Commun., 2012, 3, 1057.

16 F. Lei, Y. Sun, K. Liu, S. Gao, L. Liang, B. Pan and Y. Xie, J. Am. Chem. Soc., 2014, 136, 6826-6829.

17 R. De Levie, J. Electroanal. Chem., 1999, 476, 92-93.

18 S. Cobo, J. Heidkamp, P.-A. Jacques, J. Fize, V. Fourmond, L. Guetaz, B. Jousselme, V. Ivanova, H. Dau and S. Palacin, Nat. Mater., 2012, 11, 802-807.

19 C. C. McCrory, S. Jung, I. M. Ferrer, S. M. Chatman, J. C. Peters and T. F. Jaramillo, J. Am. Chem. Soc., 2015, 137, 4347-4357.

20 V. Nicolosi, M. Chhowalla, M. G. Kanatzidis, M. S. Strano and J. N. Coleman, Science, 2013, 340, 1226419.

21 L. Yang, H. Hong, Q. Fu, Y. Huang, J. Zhang, X. Cui, Z. Fan, K. Liu and B. Xiang, ACS Nano, 2015, 9, 64786483.
22 M. R. Gao, M. K. Chan and Y. Sun, Nat. Commun., 2015, 6, 7493.

23 D. Voiry, H. Yamaguchi, J. Li, R. Silva, D. C. Alves, T. Fujita, M. Chen, T. Asefa, V. B. Shenoy and G. Eda, Nat. Mater., 2013, 12, 850-855.

24 X. Huang, Z. Zeng and H. Zhang, Chem. Soc. Rev., 2013, 42, 1934-1946.

25 M. S. Faber and S. Jin, Energy Environ. Sci., 2014, 7, 35193542.

26 M. Zeng and Y. Li, J. Mater. Chem. A, 2015, 3, 1494214962.

27 P. C. Vesborg, B. Seger and I. Chorkendorff, J. Phys. Chem. Lett., 2015, 6, 951-957.

28 R. Kappera, D. Voiry, S. E. Yalcin, B. Branch, G. Gupta, A. D. Mohite and M. Chhowalla, Nat. Mater., 2014, 13, 1128-1134.

29 K. Xu, F. Wang, Z. Wang, X. Zhan, Q. Wang, Z. Cheng, M. Safdar and J. He, ACS Nano, 2014, 8, 8468-8476.

30 K. Xu, Z. Wang, X. Du, M. Safdar, C. Jiang and J. He, Nanotechnology, 2013, 24, 465705.

31 Q. H. Wang, K. Kalantar-Zadeh, A. Kis, J. N. Coleman and M. S. Strano, Nat. Nanotechnol., 2012, 7, 699-712.

32 K. F. Mak, C. Lee, J. Hone, J. Shan and T. F. Heinz, Phys. Rev. Lett., 2010, 105, 136805.

33 F. Wang, Z. Wang, Q. Wang, F. M. Wang, L. Yin, K. Xu, Y. Huang and J. He, Nanotechnology, 2015, 26, 292001.

34 B. Peng, P. K. Ang and K. P. Loh, Nano Today, 2015, 10, 128-137.

35 B. Hinnemann, P. G. Moses, J. Bonde, K. P. Jørgensen, J. H. Nielsen, S. Horch, I. Chorkendorff and J. K. Nørskov, J. Am. Chem. Soc., 2005, 127, 5308-5309.

36 T. F. Jaramillo, K. P. Jørgensen, J. Bonde, J. H. Nielsen, S. Horch and I. Chorkendorff, Science, 2007, 317, 100-102.

37 Y. Feng, T. He and N. Alonso-Vante, Electrochim. Acta, 2009, 54, 5252-5256.

38 D. Susac, L. Zhu, M. Teo, A. Sode, K. Wong, P. Wong, R. Parsons, D. Bizzotto, K. Mitchell and S. Campbell, J. Phys. Chem. C, 2007, 111, 18715-18723.

39 L. Zhu, M. Teo, P. Wong, K. Wong, I. Narita, F. Ernst, K. Mitchell and S. Campbell, Appl. Catal., A, 2010, 386, 157-165.

40 D. Kong, J. J. Cha, H. Wang, H. R. Lee and Y. Cui, Energy Environ. Sci., 2013, 6, 3553-3558.

41 D. Kong, H. Wang, Z. Lu and Y. Cui, J. Am. Chem. Soc., 2014, 136, 4897-4900.

42 M.-R. Gao, Y.-F. Xu, J. Jiang, Y.-R. Zheng and S.-H. Yu, J. Am. Chem. Soc., 2012, 134, 2930-2933.

43 Y. Liu, H. Cheng, M. Lyu, S. Fan, Q. Liu, W. Zhang, Y. Zhi, C. Wang, C. Xiao, S. Wei, B. Ye and Y. Xie, J. Am. Chem. Soc., 2014, 136, 15670-15675.

44 M. R. Gao, X. Cao, Q. Gao, Y. F. Xu, Y.-R. Zheng, J. Jiang and S. H. Yu, ACS Nano, 2014, 8, 3970-3978.

45 Y. R. Zheng, M. R. Gao, Q. Gao, H. H. Li, J. Xu, Z. Y. Wu and S. H. Yu, Small, 2015, 11, 182-188.

46 F. A. Rasmussen and K. S. Thygesen, J. Phys. Chem. C, 2015, 119, 13169-13183. 
47 H. Jiang, J. Phys. Chem. C, 2012, 116, 7664-7671.

48 H. L. Zhuang and R. G. Hennig, J. Phys. Chem. C, 2013, 117, 20440-20445.

49 J. Ran, J. Zhang, J. Yu, M. Jaroniec and S. Z. Qiao, Chem. Soc. Rev., 2014, 43, 7787-7812.

50 K. Chang, Z. Mei, T. Wang, Q. Kang, S. Ouyang and J. Ye, ACS Nano, 2014, 8, 7078-7087.

51 L. Yang, D. Zhong, J. Zhang, Z. Yan, S. Ge, P. Du, J. Jiang, D. Sun, X. Wu and Z. Fan, ACS Nano, 2014, 8, 6979-6985.

52 Y. Hou, A. B. Laursen, J. Zhang, G. Zhang, Y. Zhu, X. Wang, S. Dahl and I. Chorkendorff, Angew. Chem., Int. Ed., 2013, 52, 3621-3625.

53 X. Zong, J. Han, G. Ma, H. Yan, G. Wu and C. Li, J. Phys. Chem. C, 2011, 115, 12202-12208.

54 H. W. Kroto, J. R. Heath, S. C. O’Brien, R. F. Curl and R. E. Smalley, Nature, 1985, 318, 162-163.

55 A. K. Geim, Science, 2009, 324, 1530-1534.

56 C. Rao and A. Nag, Eur. J. Inorg. Chem., 2010, 4244-4250.

57 H.-L. Tsai, J. Heising, J. L. Schindler, C. R. Kannewurf and M. G. Kanatzidis, Chem. Mater., 1997, 9, 879-882.

58 K. Novoselov, D. Jiang, F. Schedin, T. Booth, V. Khotkevich, S. Morozov and A. Geim, Proc. Natl. Acad. Sci. U. S. A., 2005, 102, 10451-10453.

59 R. Tenne, J. Mater. Res., 2006, 21, 2726-2743.

60 J. N. Coleman, M. Lotya, A. O'Neill, S. D. Bergin, P. J. King, U. Khan, K. Young, A. Gaucher, S. De and R. J. Smith, Science, 2011, 331, 568-571K.

61 G. Cunningham, M. Lotya, C. S. Cucinotta, S. Sanvito, S. D. Bergin, R. Menzel, M. S. Shaffer and J. N. Coleman, ACS Nano, 2012, 6, 3468-3480.

62 K. G. Zhou, N. N. Mao, H. X. Wang, Y. Peng and H. L. Zhang, Angew. Chem., Int. Ed., 2011, 50, 10839-10842.

63 M. A. Lukowski, A. S. Daniel, C. R. English, F. Meng, A. Forticaux, R. J. Hamers and S. Jin, Energy Environ. Sci., 2014, 7, 2608-2613.

64 J. N. Coleman, Adv. Funct. Mater., 2009, 19, 3680-3695.

65 C. M. Hansen, Hansen solubility parameters: a user's handbook, CRC Press, 2007.

66 H. Ramakrishna Matte, A. Gomathi, A. K. Manna, D. J. Late, R. Datta, S. K. Pati and C. Rao, Angew. Chem., 2010, 122, 4153-4156.

67 A. Ambrosi, Z. Sofer and M. Pumera, Chem. Commun., 2015, 51, 8450-8453.

68 M. A. Lukowski, A. S. Daniel, F. Meng, A. Forticaux, L. Li and S. Jin, J. Am. Chem. Soc., 2013, 135, 10274-10277.

69 H. Wang, Z. Lu, S. Xu, D. Kong, J. J. Cha, G. Zheng, P.-C. Hsu, K. Yan, D. Bradshaw and F. B. Prinz, Proc. Natl. Acad. Sci. U. S. A., 2013, 110, 19701-19706.

70 D. Voiry, M. Salehi, R. Silva, T. Fujita, M. Chen, T. Asefa, V. B. Shenoy, G. Eda and M. Chhowalla, Nano Lett., 2013, 13, 6222-6227.

71 G. Eda, H. Yamaguchi, D. Voiry, T. Fujita, M. Chen and M. Chhowalla, Nano Lett., 2011, 11, 5111-5116.

72 Z. Zeng, T. Sun, J. Zhu, X. Huang, Z. Yin, G. Lu, Z. Fan, Q. Yan, H. H. Hng and H. Zhang, Angew. Chem., Int. Ed., 2012, 51, 9052-9056.
73 Z. Zeng, Z. Yin, X. Huang, H. Li, Q. He, G. Lu, F. Boey and H. Zhang, Angew. Chem., Int. Ed., 2011, 50, 11093-11097.

74 J.-H. Chen, C. Jang, S. Adam, M. Fuhrer, E. Williams and M. Ishigami, Nat. Phys., 2008, 4, 377-381.

75 G. Demazeau, J. Mater. Chem., 1999, 9, 15-18.

76 M. S. Whittingham, Curr. Opin. Solid State Mater. Sci., 1996, 1, 227-232.

77 M. K. Devaraju and I. Honma, Adv. Energy Mater., 2012, 2, 284-297.

78 D. Wang, Z. Pan, Z. Wu, Z. Wang and Z. Liu, J. Power Sources, 2014, 264, 229-234.

79 X. J. Lv, G. W. She, S. X. Zhou and Y. M. Li, RSC Adv., 2013, 3, 21231-21236.

80 S. J. Bao, C. M. Li, C.-X. Guo and Y. Qiao, J. Power Sources, 2008, 180, 676-681.

81 X. Ren, L. Pang, Y. Zhang, X. Ren, H. Fan and S. F. Liu, J. Mater. Chem. A, 2015, 3, 10693-10697.

82 Y. Xu, C. Zheng, S. Wang and Y. Hou, Electrochim. Acta, 2015, 174, 653-659.

83 J. Li, X. Zhou, Z. Xia, Z. Zhang, J. Li, Y. Ma and Y. Qu, J. Mater. Chem. A, 2015, 3, 13066-13071.

84 H. Yu, X. Yu, Y. Chen, S. Zhang, P. Gao and C. Li, Nanoscale, 2015, 7, 8731-8738.

85 D. Hou, W. Zhou, X. Liu, K. Zhou, J. Xie, G. Li and S. Chen, Electrochim. Acta, 2015, 166, 26-31.

86 B. Mahler, V. Hoepfner, K. Liao and G. A. Ozin, J. Am. Chem. Soc., 2014, 136, 14121-14127.

87 M.-R. Gao, J.-X. Liang, Y.-R. Zheng, Y.-F. Xu, J. Jiang, Q. Gao, J. Li and S.-H. Yu, Nat. Commun., 2015, 6, 5982.

88 W. Zhou, K. Zhou, D. Hou, X. Liu, G. Li, Y. Sang, H. Liu, L. Li and S. Chen, ACS Appl. Mater. Interfaces, 2014, 6, 21534-21540.

89 Q. Liu, J. Shi, J. Hu, A. M. Asiri, Y. Luo and X. Sun, ACS Appl. Mater. Interfaces, 2015, 7, 3877-3881.

90 H. Liang, L. Li, F. Meng, L. Dang, J. Zhuo, A. Forticaux, Z. Wang and S. Jin, Chem. Mater., 2015, 27, 5702-5711.

91 M. R. Gao, Y. F. Xu, J. Jiang and S. H. Yu, Chem. Soc. Rev., 2013, 42, 2986-3017.

92 Y. Qian, Adv. Mater., 1999, 11, 1101-1102.

93 W. MingáLau, J. Mater. Chem., 2005, 15, 4555-4559.

94 S. Xu, Z. Lei and P. Wu, J. Mater. Chem. A, 2015, 3, 1633716347.

95 T. N. Ye, L.-B. Lv, M. Xu, B. Zhang, K. X. Wang, J. Su, X. H. Li and J.-S. Chen, Nano Energy, 2015, 15, 335342.

96 Y. Li, H. Wang, L. Xie, Y. Liang, G. Hong and H. Dai, J. Am. Chem. Soc., 2011, 133, 7296-7299.

97 L. Liao, J. Zhu, X. Bian, L. Zhu, M. D. Scanlon, H. H. Girault and B. Liu, Adv. Funct. Mater., 2013, 23, 5326-5333.

98 M. R. Gao, W. T. Yao, H.-B. Yao and S. H. Yu, J. Am. Chem. Soc., 2009, 131, 7486-7487.

99 Y. F. Xu, M. R. Gao, Y. R. Zheng, J. Jiang and S. H. Yu, Angew. Chem., Int. Ed., 2013, 52, 8546-8550.

100 D. Kong, H. Wang, J. J. Cha, M. Pasta, K. J. Koski, J. Yao and Y. Cui, Nano Lett., 2013, 13, 1341-1347. 
101 Y. Zhang, Q. Ji, G.-F. Han, J. Ju, J. Shi, D. Ma, J. Sun, Y. Zhang, M. Li, X. Y. Lang, Y. F. Zhang and Z. F. Liu, ACS Nano, 2014, 8, 8617-8624.

102 H. Li, Q. Zhang, X. Duan, X. Wu, X. Fan, X. Zhu, X. Zhuang, W. Hu, H. Zhou and A. Pan, J. Am. Chem. Soc., 2015, 137, 5284-5287.

103 V. Klee, E. Preciado, D. Barroso, A. E. Nguyen, C. Lee, K. J. Erickson, M. Triplett, B. Davis, I.-H. Lu and S. Bobek, Nano Lett., 2015, 15, 2612-2619.

104 S. Wang, X. Wang and J. H. Warner, ACS Nano, 2015, 9, 5246-5254.

105 Y. Zhan, Z. Liu, S. Najmaei, P. M. Ajayan and J. Lou, Small, 2012, 8, 966-971.

106 Y. C. Lin, W. Zhang, J.-K. Huang, K.-K. Liu, Y.-H. Lee, C.-T. Liang, C. W. Chu and L. J. Li, Nanoscale, 2012, 4, 6637-6641.

107 J. G. Song, J. Park, W. Lee, T. Choi, H. Jung, C. W. Lee, S.-H. Hwang, J. M. Myoung, J. H. Jung and S.-H. Kim, ACS Nano, 2013, 7, 11333-11340.

108 Y. Shi, H. Li, J. I. Wong, X. Zhang, Y. Wang, H. Song and H. Y. Yang, Sci. Rep., 2015, 5, 10378.

109 D. Dumcenco, D. Ovchinnikov, K. Marinov, P. Lazic, M. Gibertini, N. Marzari, O. L. Sanchez, Y. C. Kung, D. Krasnozhon and M.-W. Chen, ACS Nano, 2015, 9, 46114620.

110 J. Shi, X. Zhang, D. Ma, J. Zhu, Y. Zhang, Z. Guo, Y. Yao, Q. Ji, X. Song and Y. Zhang, ACS Nano, 2015, 9, 40174025.

111 Y. Zhang, J. Shi, G. Han, M. Li, Q. Ji, D. Ma, Y. Zhang, C. Li, X. Lang and Y. Zhang, Nano Res., 2015, 8, 28812890.

112 Q. Fu, L. Yang, W. Wang, A. Han, J. Huang, P. Du, Z. Fan, J. Zhang and B. Xiang, Adv. Mater., 2015, 27, 4732.

113 M. Zou, J. Chen, L. Xiao, H. Zhu, T. Yang, M. Zhang and M. Du, J. Mater. Chem. A, 2015, 3, 18090.

114 K. K. Liu, W. Zhang, Y. H. Lee, Y. C. Lin, M. T. Chang, C. Y. Su, C. S. Chang, H. Li, Y. Shi and H. Zhang, Nano Lett., 2012, 12, 1538-1544.

115 M. Zhi, C. Xiang, J. Li, M. Li and N. Wu, Nanoscale, 2013, 5, 72-88.

116 Q. Feng, Y. Zhu, J. Hong, M. Zhang, W. Duan, N. Mao, J. Wu, H. Xu, F. Dong and F. Lin, Adv. Mater., 2014, 26, 2648-2653.

117 J. M. Velazquez, F. H. Saadi, A. P. Pieterick, J. M. Spurgeon, M. P. Soriaga, B. S. Brunschwig and N. S. Lewis, J. Electroanal. Chem., 2014, 716, 45-48.

118 Y. Shi, H. Li and L.-J. Li, Chem. Soc. Rev., 2015, 44, 27442756.

119 J. Mann, Q. Ma, P. M. Odenthal, M. Isarraraz, D. Le, E. Preciado, D. Barroso, K. Yamaguchi, G. von Son Palacio and A. Nguyen, Adv. Mater., 2014, 26, 1399-1404.

120 M. Zhang, J. Wu, Y. Zhu, D. O. Dumcenco, J. Hong, N. Mao, S. Deng, Y. Chen, Y. Yang and C. Jin, ACS Nano, 2014, 8, 7130-7137.

121 J. Kibsgaard, Z. Chen, B. N. Reinecke and T. F. Jaramillo, Nat. Mater., 2012, 11, 963-969.
122 M. S. Faber, R. Dziedzic, M. A. Lukowski, N. S. Kaiser, Q. Ding and S. Jin, J. Am. Chem. Soc., 2014, 136, 1005310061.

123 H. Wang, D. Kong, P. Johanes, J. J. Cha, G. Zheng, K. Yan, N. Liu and Y. Cui, Nano Lett., 2013, 13, 3426-3433.

124 F. Wang, Y. Wang, X. Zhan, M. Safdar, J. Gong and J. He, CrystEngComm, 2014, 16, 1389-1391.

125 F. Wang, X. Zhan, Z. Cheng, Z. Wang, Q. Wang, K. Xu, M. Safdar and J. He, Small, 2015, 11, 749-755.

126 K. Wang, D. Xi, C. Zhou, Z. Shi, H. Xia, G. Liu and G. Qiao, J. Mater. Chem. A, 2015, 3, 9415-9420.

127 H. Zhu, M. Du, M. Zhang, M. Zou, T. Yang, Y. Fu and J. Yao, J. Mater. Chem. A, 2014, 2, 7680-7685.

128 Y. H. Chang, C. T. Lin, T. Y. Chen, C. L. Hsu, Y. H. Lee, W. Zhang, K. H. Wei and L. J. Li, Adv. Mater., 2013, 25, 756-760.

129 Y. Tan, P. Liu, L. Chen, W. Cong, Y. Ito, J. Han, X. Guo, Z. Tang, T. Fujita and A. Hirata, Adv. Mater., 2014, 26, 8023-8028.

130 M. S. Faber, M. A. Lukowski, Q. Ding, N. S. Kaiser and S. Jin, J. Phys. Chem. C, 2014, 118, 21347-21356.

131 X. Wu, B. Yang, Z. Li, L. Lei and X. Zhang, RSC Adv., 2015, 5, 32976-32982.

132 Y. Jiao, Y. Zheng, M. Jaroniec and S. Z. Qiao, Chem. Soc. Rev., 2015, 44, 2060-2086.

133 B. Conway and B. Tilak, Electrochim. Acta, 2002, 47, 35713594.

134 Y. Zheng, Y. Jiao, M. Jaroniec and S. Z. Qiao, Angew. Chem., Int. Ed., 2015, 54, 52-65.

135 W. Sheng, H. A. Gasteiger and Y. Shao-Horn, J. Electrochem. Soc., 2010, 157, B1529.

136 R. Parsons, Trans. Faraday Soc., 1958, 54, 1053-1063.

137 V. I. Birss, A. Damjanovic and P. Hudson, J. Electrochem. Soc., 1986, 133, 1621-1625.

138 J. D. Benck, T. R. Hellstern, J. Kibsgaard, P. Chakthranont and T. F. Jaramillo, ACS Catal., 2014, 4, 3957-3971.

139 A. B. Laursen, S. Kegnæs, S. Dahl and I. Chorkendorff, Energy Environ. Sci., 2012, 5, 5577-5591.

140 S. Balendhran, S. Walia, H. Nili, J. Z. Ou, S. Zhuiykov, R. B. Kaner, S. Sriram, M. Bhaskaran and K. Kalantarzadeh, Adv. Funct. Mater., 2013, 23, 3952-3970.

141 D. Merki, S. Fierro, H. Vrubel and X. Hu, Chem. Sci., 2011, 2, 1262-1267.

142 B. Seo, G. Y. Jung, Y. J. Sa, H. Y. Jeong, J. Y. Cheon, J. H. Lee, H. Y. Kim, J. C. Kim, H. S. Shin and S. K. Kwak, ACS Nano, 2015, 9, 3728-3739.

143 D. Y. Chung, S.-K. Park, Y.-H. Chung, S.-H. Yu, D.-H. Lim, N. Jung, H. C. Ham, H.-Y. Park, Y. Piao and S. J. Yoo, Nanoscale, 2014, 6, 2131-2136.

144 J. Xie, H. Zhang, S. Li, R. Wang, X. Sun, M. Zhou, J. Zhou, X. W. D. Lou and Y. Xie, Adv. Mater., 2013, 25, 5807-5813.

145 N. R. Paudel and Y. Yan, Appl. Phys. Lett., 2014, 104, 143507.

146 F. H. Saadi, A. I. Carim, J. M. Velazquez, J. H. Baricuatro, C. C. L. McCrory, M. P. Soriaga and N. S. Lewis, ACS Catal., 2014, 4, 2866-2873. 
147 Z. Chen, D. Cummins, B. N. Reinecke, E. Clark, M. K. Sunkara and T. F. Jaramillo, Nano Lett., 2011, 11, 4168-4175.

148 Y. Yan, B. Xia, N. Li, Z. Xu, A. Fisher and X. Wang, J. Mater. Chem. A, 2015, 3, 131-135.

149 D. H. Youn, S. Han, J. Y. Kim, J. Y. Kim, H. Park, S. H. Choi and J. S. Lee, ACS Nano, 2014, 8, 5164-5173.

150 D. J. Li, U. N. Maiti, J. Lim, D. S. Choi, W. J. Lee, Y. Oh, G. Y. Lee and S. O. Kim, Nano Lett., 2014, 14, 1228-1233.

151 T. Wang, L. Liu, Z. Zhu, P. Papakonstantinou, J. Hu, H. Liu and M. Li, Energy Environ. Sci., 2013, 6, 625-633.

152 C. B. Ma, X. Qi, B. Chen, S. Bao, Z. Yin, X. J. Wu, Z. Luo, J. Wei, H.-L. Zhang and H. Zhang, Nanoscale, 2014, 6, 5624-5629.

153 S. Mao, Z. Wen, S. Ci, X. Guo, K. K. Ostrikov and J. Chen, Small, 2015, 11, 414-419.

154 Y. Huang, Y.-E. Miao, J. Fu, S. Mo, C. Wei and T. Liu, J. Mater. Chem. A, 2015, 3, 16263-16271.

155 H. Tang, K. Dou, C.-C. Kaun, Q. Kuang and S. Yang, J. Mater. Chem. A, 2014, 2, 360-364.

156 H. Wang, Z. Lu, D. Kong, J. Sun, T. M. Hymel and Y. Cui, ACS Nano, 2014, 8, 4940-4947.

157 D. Allan, A. Kelsey, S. Clark, R. Angel and G. Ackland, Phys. Rev. B: Condens. Matter, 1998, 57, 5106.

158 J. Xie, J. Zhang, S. Li, F. Grote, X. Zhang, H. Zhang, R. Wang, Y. Lei, B. Pan and Y. Xie, J. Am. Chem. Soc., 2013, 135, 17881-17888.

159 C. Xu, S. Peng, C. Tan, H. Ang, H. Tan, H. Zhang and Q. Yan, J. Mater. Chem. A, 2014, 2, 5597-5601.

160 V. Kiran, D. Mukherjee, R. N. Jenjeti and S. Sampath, Nanoscale, 2014, 6, 12856-12863.

161 Q. Gong, L. Cheng, C. Liu, M. Zhang, Q. Feng, H. Ye, M. Zeng, L. Xie, Z. Liu and Y. Li, ACS Catal., 2015, 5, 2213-2219.

162 L. Yang, Q. Fu, W. Wang, J. Huang, J. Huang, J. Zhang and B. Xiang, Nanoscale, 2015, 7, 10490-10497.

163 J. Bonde, P. G. Moses, T. F. Jaramillo, J. K. Nørskov and I. Chorkendorff, Faraday Discuss., 2009, 140, 219-231.

164 D. Merki, H. Vrubel, L. Rovelli, S. Fierro and X. Hu, Chem. Sci., 2012, 3, 2515-2525.

165 C. Tsai, K. Chan, F. Abild-Pedersen and J. K. Nørskov, Phys. Chem. Chem. Phys., 2014, 16, 13156-13164.

166 A. Sobczynski, A. Yildiz, A. J. Bard, A. Campion, M. A. Fox, T. Mallouk, S. E. Webber and J. M. White, J. Phys. Chem., 1988, 92, 2311-2315.

167 L. Cheng, W. Huang, Q. Gong, C. Liu, Z. Liu, Y. Li and H. Dai, Angew. Chem., Int. Ed., 2014, 53, 7860-7863.

168 J. Lin, Z. Peng, G. Wang, D. Zakhidov, E. Larios, M. J. Yacaman and J. M. Tour, Adv. Energy Mater., 2014, 4, 1301875-1301882.

169 R. Tenne, L. Margulis, M. e. a. Genut and G. Hodes, Nature, 1992, 360, 444-446.

170 M. Remikar, Z. Škraba, M. Regula, C. Ballif, R. Sanjinés and F. Lévy, Adv. Mater., 1998, 10, 246-249.

171 L. Margulis, G. Salitra, R. Tenne and M. Tallanker, Nature, 1993, 365, 113-114.
172 F. Wang, J. Li, F. Wang, T. A. Shifa, Z. Cheng, Z. Wang, K. Xu, X. Zhan, Q. Wang, Y. Huang, C. Jiang and J. He, Adv. Funct. Mater., 2015, 25, 6077.

173 J. Yang, D. Voiry, S. J. Ahn, D. Kang, A. Y. Kim, M. Chhowalla and H. S. Shin, Angew. Chem., Int. Ed., 2013, 52, 13751-13754.

174 T. A. Shifa, F. Wang, Z. Cheng, X. Zhan, Z. Wang, K. Liu, S. Muhammad, L. Sun and J. He, Nanoscale, 2015, 7, 14760-14765.

175 L. Wang, Z. Sofer, J. Luxa and M. Pumera, Adv. Mater. Interfaces, 2015, 2, 1500041-1500045.

176 J. Kushmerick, S. Kandel, P. Han, J. Johnson and P. Weiss, J. Phys. Chem. B, 2000, 104, 2980-2988.

177 B. R. Leliveld, J. A. van Dillen, J. W. Geus, D. C. Koningsberger and M. de Boer, J. Phys. Chem. B, 1997, 101, 11160-11171.

178 A. Sobczynski, A. Bard, A. Campion, M. Fox, T. Mallouk, S. Webber and J. White, J. Phys. Chem., 1989, 93, 401403.

179 C. H. Lin, C. L. Chen and J.-H. Wang, J. Phys. Chem. C, 2011, 115, 18582-18588.

180 H. Zhang, Y. Li, G. Zhang, T. Xu, P. Wan and X. Sun, J. Mater. Chem. A, 2015, 3, 6306-6310.

181 K. Wang, D. Xi, C. Zhou, Z. Shi, H. Xia, G. Liu and G. Qiao, J. Mater. Chem. A, 2015, 3, 9415-9420.

182 N. Kornienko, J. Resasco, N. Becknell, C.-M. Jiang, Y.-S. Liu, K. Nie, X. Sun, J. Guo, S. R. Leone and P. Yang, J. Am. Chem. Soc., 2015, 137, 7448-7455.

183 M. S. Faber, M. A. Lukowski, Q. Ding, N. S. Kaiser and S. Jin, J. Phys. Chem. C, 2014, 118, 21347-21356.

184 H. Zhang, B. Yang, X. Wu, Z. Li, L. Lei and X. Zhang, ACS Appl. Mater. Interfaces, 2015, 7, 1772-1779.

185 D. Y. Wang, M. Gong, H. L. Chou, C. J. Pan, H. A. Chen, Y. Wu, M. C. Lin, M. Guan, J. Yang, C. W. Chen, Y. L. Wang, B. J. Hwang, C. C. Chen and H. Dai, J. Am. Chem. Soc., 2015, 137, 1587-1592.

186 M. Caban-Acevedo, M. L. Stone, J. R. Schmidt, J. G. Thomas, Q. Ding, H.-C. Chang, M.-L. Tsai, J. H. He and S. Jin, Nat. Mater., 2015, DOI: 10.1038/nmat4410.

187 J. Suntivich, K. J. May, H. A. Gasteiger, J. B. Goodenough and Y. Shao-Horn, Science, 2011, 334, 1383-1385.

188 L. Wu, Q. Li, C. H. Wu, H. Zhu, A. Mendoza-Garcia, B. Shen, J. Guo and S. Sun, J. Am. Chem. Soc., 2015, 137, 7071-7074.

189 T.-L. Wee, B. D. Sherman, D. Gust, A. L. Moore, T. A. Moore, Y. Liu and J. C. Scaiano, J. Am. Chem. Soc., 2011, 133, 16742-16745.

190 N. H. Chou, P. N. Ross, A. T. Bell and T. D. Tilley, ChemSusChem, 2011, 4, 1566-1569.

191 B. S. Yeo and A. T. Bell, J. Am. Chem. Soc., 2011, 133, 5587-5593.

192 R. D. Smith, M. S. Prévot, R. D. Fagan, Z. Zhang, P. A. Sedach, M. K. J. Siu, S. Trudel and C. P. Berlinguette, Science, 2013, 340, 60-63.

193 Y. Li, P. Hasin and Y. Wu, Adv. Mater., 2010, 22, 19261929. 
194 L. Trotochaud, J. K. Ranney, K. N. Williams and S. W. Boettcher, J. Am. Chem. Soc., 2012, 134, 1725317261.

195 T. Y. Ma, S. Dai, M. Jaroniec and S. Z. Qiao, J. Am. Chem. Soc., 2014, 136, 13925-13931.

196 M. Risch, K. A. Stoerzinger, S. Maruyama, W. T. Hong, I. Takeuchi and Y. Shao-Horn, J. Am. Chem. Soc., 2014, 136, 5229-5232.

197 R. Subbaraman, D. Tripkovic, K.-C. Chang, D. Strmcnik, A. P. Paulikas, P. Hirunsit, M. Chan, J. Greeley, V. Stamenkovic and N. M. Markovic, Nat. Mater., 2012, 11, 550-557.

198 M. W. Kanan and D. G. Nocera, Science, 2008, 321, 10721075.

199 D. K. Zhong and D. R. Gamelin, J. Am. Chem. Soc., 2010, 132, 4202-4207.

200 E. J. Popczun, C. G. Read, C. W. Roske, N. S. Lewis and R. E. Schaak, Angew. Chem., 2014, 126, 5531-5534.

201 K. J. May, C. E. Carlton, K. A. Stoerzinger, M. Risch, J. Suntivich, Y. L. Lee, A. Grimaud and Y. Shao-Horn, J. Phys. Chem. Lett., 2012, 3, 3264-3270.

202 M. R. Gao, Q. Gao, J. Jiang, C. H. Cui, W. T. Yao and S. H. Yu, Angew. Chem., 2011, 123, 5007-5010.

203 H. Tang, H. Yin, J. Wang, N. Yang, D. Wang and Z. Tang, Angew. Chem., 2013, 125, 5695-5699.

204 H. Jin, J. Wang, D. Su, Z. Wei, Z. Pang and Y. Wang, J. Am. Chem. Soc., 2015, 137, 2688-2694.

205 B. Hammer and J. K. Nørskov, Adv. Catal., 2000, 45, 71-129.

206 P. Ganesan, M. Prabu, J. Sanetuntikul and S. Shanmugam, ACS Catal., 2015, 5, 3625-3637.

207 S. Y. Reece, J. A. Hamel, K. Sung, T. D. Jarvi, A. J. Esswein, J. J. Pijpers and D. G. Nocera, Science, 2011, 334, 645-648.

208 A. Fujishima, Nature, 1972, 238, 37-38.

209 K. Maeda, K. Teramura, D. Lu, T. Takata, N. Saito, Y. Inoue and K. Domen, Nature, 2006, 440, 295-295.

210 A. Kudo and Y. Miseki, Chem. Soc. Rev., 2009, 38, 253-278.

211 X. Chen, S. Shen, L. Guo and S. S. Mao, Chem. Rev., 2010, 110, 6503-6570.

212 J. Yang, H. Yan, X. Zong, F. Wen, M. Liu and C. Li, Philos. Trans. R. Soc. London, Ser. A, 2013, 371, 20110430.

213 F. E. Osterloh, Chem. Mater., 2008, 20, 35-54.

214 W. Ho, J. C. Yu, J. Lin, J. Yu and P. Li, Langmuir, 2004, 20, 5865-5869.

215 H. Abruna, G. Hope and A. Bard, J. Electrochem. Soc., 1982, 129, 2224-2228.

216 H. Tributsch and J. Bennett, J. Electroanal. Chem., 1977, 81, 97-111.

217 N. Singh, G. Jabbour and U. Schwingenschlögl, Eur. Phys. J. B, 2012, 85, 1-4.

218 R. Neville and B. Evans, Phys. Status Solidi B, 1976, 73, 597-606.

219 C. D. Spataru, S. Ismail-Beigi, L. X. Benedict and S. G. Louie, Phys. Rev. Lett., 2004, 92, 077402.

220 A. Splendiani, L. Sun, Y. Zhang, T. Li, J. Kim, C.-Y. Chim, G. Galli and F. Wang, Nano Lett., 2010, 10, 1271-1275.
221 Z. Zhu, Y. Cheng and U. Schwingenschlögl, Phys. Rev. B: Condens. Matter Mater. Phys., 2011, 84, 153402.

222 X. Zong, Y. Na, F. Wen, G. Ma, J. Yang, D. Wang, Y. Ma, M. Wang, L. Sun and C. Li, Chem. Commun., 2009, 45364538.

223 Y. Li, Y.-L. Li, C. M. Araujo, W. Luo and R. Ahuja, Catal. Sci. Technol., 2013, 3, 2214-2220.

224 D. Merki and X. Hu, Energy Environ. Sci., 2011, 4, 38783888.

225 S. Bai, L. Wang, X. Chen, J. Du and Y. Xiong, Nano Res., 2015, 8, 175-183.

226 Q. Xiang, J. Yu and M. Jaroniec, J. Am. Chem. Soc., 2012, 134, 6575-6578.

227 F. A. Frame and F. E. Osterloh, J. Phys. Chem. C, 2010, 114, 10628-10633.

228 Y. P. Yuan, S.-W. Cao, L.-S. Yin, L. Xu and C. Xue, Int. J. Hydrogen Energy, 2013, 38, 7218-7223.

229 J. Chen, X. J. Wu, L. Yin, B. Li, X. Hong, Z. Fan, B. Chen, C. Xue and H. Zhang, Angew. Chem., Int. Ed., 2015, 54, 1210-1214.

230 B. Pourabbas and B. Jamshidi, Chem. Eng. J., 2008, 138, $55-62$.

231 K. H. Hu, X. G. Hu, Y. F. Xu and J. D. Sun, J. Mater. Sci., 2010, 45, 2640-2648.

232 I. Tacchini, E. Terrado, A. Anson and M. Martínez, Micro Nano Lett., 2011, 6, 932-936.

233 W. Li, Y. Y. Sara and E. Iglesia, J. Catal., 2002, 207, $31-36$.

234 S. J. Moniz, S. A. Shevlin, D. J. Martin, Z.-X. Guo and J. Tang, Energy Environ. Sci., 2015, 8, 731-759.

235 N. Bao, L. Shen, T. Takata and K. Domen, Chem. Mater., 2008, 20, 110-117.

236 X. Wang, K. Maeda, A. Thomas, K. Takanabe, G. Xin, J. M. Carlsson, K. Domen and M. Antonietti, Nat. Mater., 2009, 8, 76-80.

237 X. Zong, H. Yan, G. Wu, G. Ma, F. Wen, L. Wang and C. Li, J. Am. Chem. Soc., 2008, 130, 7176-7177.

238 M. Nguyen, P. D. Tran, S. S. Pramana, R. L. Lee, S. K. Batabyal, N. Mathews, L. H. Wong and M. Graetzel, Nanoscale, 2013, 5, 1479-1482.

239 M. Latorre-Sánchez, I. Esteve-Adell, A. Primo and H. García, Carbon, 2015, 81, 587-596.

240 P. D. Tran, S. S. Pramana, V. S. Kale, M. Nguyen, S. Y. Chiam, S. K. Batabyal, L. H. Wong, J. Barber and J. Loo, Chem.-Eur. J., 2012, 18, 13994-13999.

241 B. Seger, A. B. Laursen, P. C. Vesborg, T. Pedersen, O. Hansen, S. Dahl and I. Chorkendorff, Angew. Chem., Int. Ed., 2012, 51, 9128-9131.

242 Q. Ding, F. Meng, C. R. English, M. Cabán-Acevedo, M. J. Shearer, D. Liang, A. S. Daniel, R. J. Hamers and S. Jin, J. Am. Chem. Soc., 2014, 136, 8504-8507.

243 M. W. Knight, H. Sobhani, P. Nordlander and N. J. Halas, Science, 2011, 332, 702-704.

244 J. S. DuChene, B. C. Sweeny, A. C. Johnston-Peck, D. Su, E. A. Stach and W. D. Wei, Angew. Chem., Int. Ed., 2014, 53, 7887-7891. 
245 Z. Bian, T. Tachikawa, P. Zhang, M. Fujitsuka and T. Majima, J. Am. Chem. Soc., 2014, 136, 458-465.

246 R. Su, R. Tiruvalam, A. J. Logsdail, Q. He, C. A. Downing, M. T. Jensen, N. Dimitratos, L. Kesavan, P. P. Wells and R. Bechstein, ACS Nano, 2014, 8, 3490-3497.

247 Y. Kang, S. Najmaei, Z. Liu, Y. Bao, Y. Wang, X. Zhu, N. J. Halas, P. Nordlander, P. M. Ajayan, J. Lou and Z. Fang, Adv. Mater., 2014, 26, 6467-6471.

248 Y. Shi, J. Wang, C. Wang, T.-T. Zhai, W.-J. Bao, J.-J. Xu, X.-h. Xia and H.-Y. Chen, J. Am. Chem. Soc., 2015, 137, 7365-7370.
249 Y. Kang, Y. Gong, Z. Hu, Z. Li, Z. Qiu, X. Zhu, P. M. Ajayan and Z. Fang, Nanoscale, 2015, 7, 4482-4488.

250 Z. Yin, B. Chen, M. Bosman, X. Cao, J. Chen, B. Zheng and H. Zhang, Small, 2014, 10, 3537-3543.

251 F. A. Rasmussen and K. S. Thygesen, J. Phys. Chem. C, 2015, 119, 13169-13183.

252 H. Jiang, J. Phys. Chem. C, 2012, 116, 7664-7671.

253 B. Mahler, V. Hoepfner, K. Liao and G. A. Ozin, J. Am. Chem. Soc., 2014, 136, 14121-14127.

254 G. Gao, Y. Jiao, F. Ma, Y. Jiao, E. Waclawik and A. Du, J. Phys. Chem. C, 2015, 119, 13124-13128. 